\title{
Mechanisms of Compound Kushen Injection for Treatment of Bladder Cancer Based on Bioinformatics and Network Pharmacology With Experimental Validation
}

\section{Lihui Zhang \\ hebeidaxue \\ Wanying Zhang \\ Hebei University \\ Jiaming Xiong \\ Hebei University \\ Xiumei Duan}

Beijing Zhendong Guangming Pharmaceutical Research Institute Co Ltd

Lina Hai

Beijing Zhendong Guangming Pharmaceutical Research Co Ltd

\section{Yuliang Zhang}

Hebei University

Miaomiao Zhang

Hebei University

\section{Guifang Qin}

Hebei University

Guowei Zhang ( $\nabla x x z$ w@126.com )

Hebei University

\section{Research}

Keywords: Compound Kushen Injection, bladder cancer, network pharmacology, GEO, molecular mechanism

Posted Date: September 8th, 2020

DOl: https://doi.org/10.21203/rs.3.rs-71493/v1

License: (c) (i) This work is licensed under a Creative Commons Attribution 4.0 International License. 
Version of Record: A version of this preprint was published at Chinese Journal of Natural Medicines on January 1st, 2022. See the published version at https://doi.org/10.1016/S1875-5364(22)60144-4. 


\title{
Mechanisms of Compound Kushen Injection for Treatment of Bladder Cancer Based on Bioinformatics and Network Pharmacology with Experimental Validation
}

\author{
Lihui Zhang ${ }^{1}$, Wanying Zhang ${ }^{1}$, Jiaming Xiong ${ }^{1}$, Xiumei Duan ${ }^{2}$, Lina Hai ${ }^{2,3}$, Yuliang Zhang ${ }^{1}$, \\ Miaomiao Zhang ${ }^{1}$, Guifang Qin ${ }^{1}$, Guowei Zhang ${ }^{*}$
}

\begin{abstract}
Background: Bladder cancer is the most common malignant neoplasm of the urinary system. Compound Kushen injection (CKI) is a Chinese medicinal preparation that has been used clinically to treat various types of cancers for more than 20 years. However, the pharmacological effect of CKI on bladder cancer requires further clarification.
\end{abstract}

Methods: Network pharmacology combined with bioinformatics was used to elucidate the therapeutic mechanism and potential targets of CKI in bladder cancer. The mechanism by which CKI is effective against bladder cancer was further verified in vitro using the human bladder cancer cell line T24.

Results: Network pharmacology analysis identified 35 active compounds and 268 target genes of CKI. Bioinformatics data mining revealed 5500 differentially expressed genes associated with bladder cancer. Common genes of CKI and bladder cancer suggested that CKI exerts anti-bladder cancer effects by regulating genes such as MMP-9, JUN, EGFR, and ERK1. Functional enrichment analysis indicated that CKI has a therapeutic effect on bladder cancer by synergistically regulating certain biological processes, including cell proliferation, cell migration, and cell apoptosis. In addition, Kyoto Encyclopedia of Genes and Genomes enrichment analysis implicated pathways related to cancer, bladder cancer, and the PI3K-Akt signaling pathway. Consistently, cell experiments indicated that CKI could inhibit proliferation and migration of T24 bladder cancer cells, and induce their apoptosis. Moreover, RT-qPCR and western blot results indicated that CKI may treat bladder cancer by downregulating gene and protein expression levels, respectively of MMP-9, JUN, EGFR, and ERK1.

Conclusions: CKI can inhibit proliferation and migration, and induce apoptosis of T24 bladder cancer cells through multiple biological pathways and targets. CKI also has significant effects on regulation of key genes and proteins associated with bladder cancer. Overall, our findings provide solid evidence and deepen current understanding of the therapeutic effects of CKI for bladder cancer, and further support its clinical use.

Keywords: Compound Kushen Injection, bladder cancer, network pharmacology, GEO, molecular mechanism

\footnotetext{
* Correspondence: xxzgw@126.com

${ }^{1}$ College of Traditional Chinese Medicine, Hebei University, Baoding, Hebei, 071002, China

Full list of author information is available at the end of the article
} 


\section{Background}

With an estimated 549,000 cases and 200,000 deaths annually, bladder cancer was the 10th most frequently diagnosed cancer worldwide in 2018. Globally, the incidence rate of this disease is higher among men, which is at about four times that of women [1]. Approximately $75 \%$ of cases were diagnosed as non-muscle-invasive disease (NMIBC), while the remaining $25 \%$ were diagnosed as muscle-invasive bladder cancer (MIBC) [2]. Environmental factors, such as cigarette smoking and long-term exposure to chemical or water contaminants, can increase the risk of bladder cancer [3]. Current therapy for NMIBC is transurethral resection, while radical cystectomy is usually prescribed for MIBC. Chemotherapy, radiotherapy, immunotherapy, and targeted therapy are also commonly used for bladder cancer [4]. Despite these advanced treatments for bladder cancer, the local recurrence rate and distant metastasis rate of patients remain high [5]. Therefore, it is important to identify new strategies and treatment methods to improve the survival rate of patients with bladder cancer [6].

Traditional Chinese Medicine (TCM) has been widely used to treat various types of cancers and has showed great therapeutic effects in both scientific research and the clinic [7]. As a result of reliable efficacy and few adverse reactions, TCM has gained popularity around the world [8]. Compound Kushen injection (CKI), a TCM preparation refined from Sophora flavescens Aiton and Heterosmilax japonica Kunth, has been clinically ratified in China for more than 20 years [9]. CKI is often used to treat cancers of the lung, liver, and breast, as well as gastric carcinoma, multiple myeloma, and other cancers [10]. Previous reports have shown that $\mathrm{CKI}$ has multiple effects; not only can CKI reduce cancer pain and hemorrhage, it can also diminish inflammation and enhance the body's immunity [11]. In addition, CKI sensitizes cancer cells to chemotherapeutic drugs, reduces the side effects of chemotherapy, and improves the quality of life in patients with cancer [12]. Furthermore, CKI can stimulate anti-cancer activities by inhibiting cancer cell proliferation, causing apoptosis, perturbing the cell cycle, preventing tumor cell migration and invasion, and interfering in other cancer signaling pathways [13, 14]. Importantly, in the routine treatment of bladder cancer, the application of CKI can improve the efficacy and reduce the adverse reactions caused by chemotherapy, and reduce the recurrence rate. Therefore, application of CKI adjuvant treatment for bladder cancer may be a breakthrough to solve problems associated with current therapeutic strategies.

In recent years, network pharmacology has emerged as a useful tool to explain the regulatory effects of TCM on the human body from a systemic perspective and at a molecular level [15]. TCM exploits the characteristics of "multi-component, multi-pathway and multi-target" synergistic effects, thus making it possible to treat various complex diseases. With the rise of network pharmacology, it is possible for us to discover TCM from a holistic and molecular level to reveal the molecular mechanisms of active ingredients in Chinese medicinal herbs [16].

With the help of bioinformatics, differentially expressed genes (DEGs) between bladder cancer and normal tissues from Gene Expression Omnibus (GEO) datasets can identify and improve our understanding of significant biomarkers [17]. In this study, network pharmacology was first used to investigate effective components and potential targets to predict the molecular mechanisms of CKI against bladder cancer. Next, in vitro experiments using human bladder cancer T24 cells were used to verify the main molecular mechanism of CKI in treating bladder cancer (Fig. 1). 

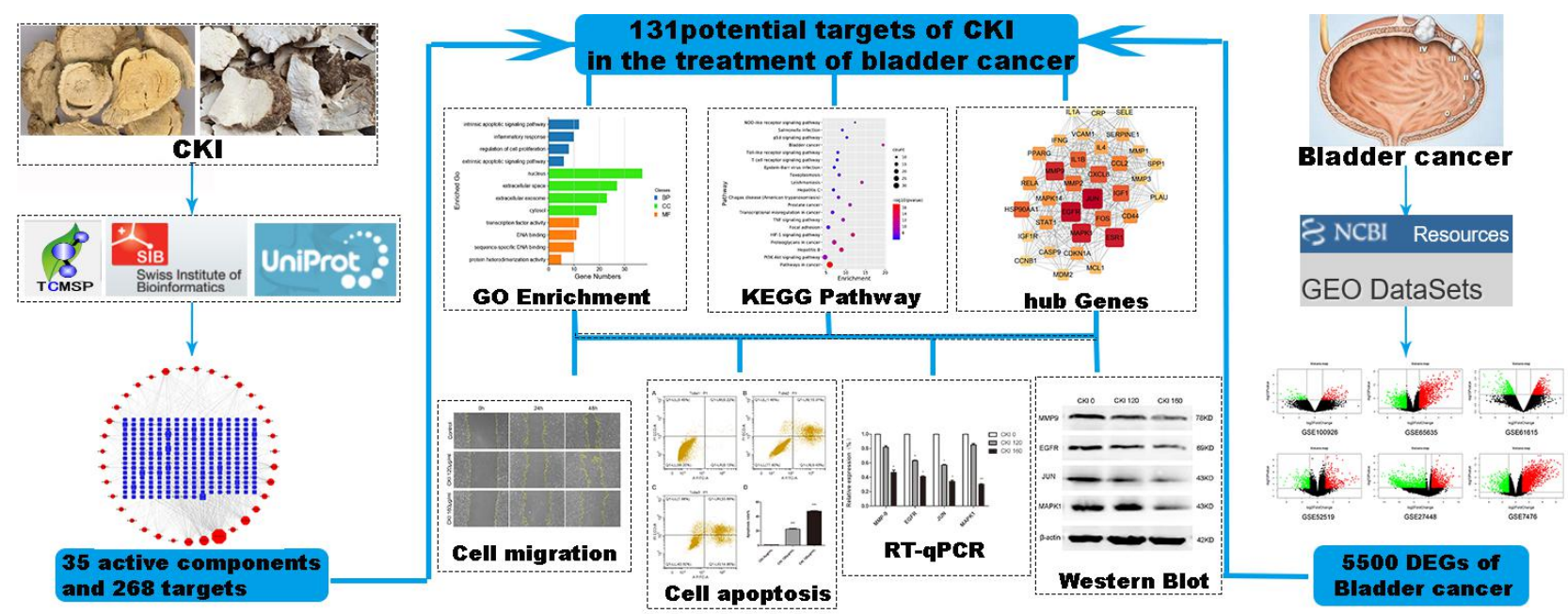

Fig. 1 Scheme of the study

\section{Methods}

\section{Screening of active ingredients of CKI}

Traditional Chinese Medicine Systems Pharmacology (TCMSP, http://tcmspw.com/ tcmsp.php), a unique pharmacology platform for Chinese herbal medicine, can explain the mechanism of small molecules and compounds of TCM to help realize its modernization [18]. Constituents of the two herbal components of CKI were retrieved from the TCMSP database, but Heterosmilax yunnanensis Gagnep was not included. Therefore, active ingredients of Sophora flavescens Aiton were searched through TCMSP, while active ingredients of Heterosmilax yunnanensis Gagnep were collected according to reported literature. The active components of CKI were screened by applying absorption, distribution, metabolism, and excretion conditions, and Lipinski's rule as follows: drug likelihood (DL) $\geq$ 0.18 , hydrogen bond acceptors (Hacc) $\leq 10$, hydrogen bond donors (Hdon) $\leq 5$, and octanol-water partition $(\log \mathrm{P}) \leq 5[19,20]$.

Target prediction for bioactive ingredients of CKI

To identify target genes of active compounds in CKI, TCMSP database and SwissTargetPrediction (http://www. Swisstarget prediction.ch/) were used to retrieve potential targets. In the present study, a confidence score $\geq 0.9$ was used as the inclusion criteria [21]. Obtained target information was normalized using the UniProt database (https://www.uniprot.org/), and targets for nonhuman source species were deleted.

\section{Screening of differentially expressed genes in bladder cancer}

The GEO database (https://www.ncbi.nlm.nih. gov/geo/), a gene expression database created and maintained by the National Center for Biotechnology Information, contains highthroughput gene expression data submitted by research institutions around the world. We used "bladder cancer" as the key word to search the GEO database and collect all gene chip data; original expression matrix was downloaded. After standardization of multiple GEO data, the limma package for R software was applied to screen DEGs between normal and bladder cancer tissues [22]. DEGs with a $\mathrm{P}$ value $<0.05$ and $|\operatorname{logFC}|>2$ were considered significantly upregulated, while DEGs with a $\mathrm{P}$ value $<0.05$ and $|\log \mathrm{FC}|>-2$ were considered significantly downregulated. Volcano plots of screened significantly DEGs were made using the ggplot2 package for R.

Construction of drug active ingredients and disease target network

Targets of CKI and DEGs of bladder cancer were 
intersected and visualized by Venny diagram (http://bioinfogp.cnb.csic.es/tools/venny/index.html) . Common genes were considered potential targets of CKI for the treatment of bladder cancer. Next, results for active components of CKI and common targets of CKI and bladder cancer were imported into Cytoscape (v3.7.2, http://www.cytoscape.org) [23] to construct a "component-target-disease" network. Nodes in the network represent chemical components, while targets and the correlation between the component and target are represented by edges. To analyze the main active ingredients of CKI involved in the treatment of bladder cancer, features such as degree, closeness centrality, and betweenness centrality were evaluated using the "network analyzer" tool.

\section{Enrichment analyses}

Database Visualization and Integrated Discovery software (DAVID, version 6.8; https:// david.ncifcrf.gov/) [24] was used for Gene Ontology (GO) enrichment analysis [25] and Kyoto Encyclopedia of Genes and Genomes (KEGG) pathway analysis [26]. The main pathway and biological functions of CKI against bladder cancer were screened according to false discovery rate value, and the results were visualized with $R$ software.

\section{PPI network analysis}

Constructing a protein-protein interaction (PPI) network can facilitate understanding of the core regulatory genes of CKI with regard to the treatment of bladder cancer and reveal in-depth mechanisms of disease occurrence and development [27]. Common targets of CKI and bladder cancer were imported into the STRING database (https:// string-db.org/), a protein interaction database with the most covered species and largest amount of interaction information. After setting the species to Homo sapiens, we screened the interaction target network with a comprehensive score greater than 0.4 and imported the resulting TSV file into Cytoscape software. Previous studies have showen that Molecular Complex Detection (MCODE) is the most reliable gene network module analysis algorithm for protein complex detection [28]. A highly interconnected module in the PPI network was identified using the MCODE plugin of Cytoscape and visualized by Cytoscape. Screening criteria included: degree cutoff $=2$, node score cutoff $=0.2, \mathrm{~K}$-score $=10$ and max.depth $=100$. Proteins in this module were considered core targets of CKI for the treatment of bladder cancer.

\section{Cell culture}

The human bladder cancer cell line T24 was obtained from the Cell Bank of the Chinese Academy of Sciences. Cells were cultured in McCoy's 5A medium with $10 \%$ fetal bovine serum (Gibco Company, Grand Island, NY), 100 units/mL penicillin, and $100 \mu \mathrm{g} / \mathrm{mL}$ streptomycin in a humidified atmosphere of $5 \% \mathrm{CO}_{2}$ at $37{ }^{\circ} \mathrm{C}$.

\section{Cell viability assay}

A Cell Counting Kit 8 (CCK-8; Dojin Laboratories, Kumamoto, Japan) was used to examine the proliferative ability of T24 cells. T24 cells were seeded at a density of $8 \times 10^{3}$ cells/well in $100 \mu \mathrm{L}$ of medium in a 96-well plate (Costar Corning, Rochester, NY) and treated with different concentrations $(0,40,80,120$, or $160 \mu \mathrm{g} / \mathrm{mL})$ of CKI (Batch number: 20171122, Zhendong pharmaceutical, Shanxi) for 12, 24, and $48 \mathrm{~h}$. Next, $10 \mu \mathrm{L}$ of CCK-8 working solution was added to each well for 2-h incubation in the incubator. Absorbance was measured with a microplate reader (BioTek Instruments, Winooski, VT) at a 490-nm wavelength. According to $\mathrm{IC}_{50}$ values evaluated for each time period, we ultimately chose 0,120 , and $160 \mu \mathrm{g} / \mathrm{mL} \mathrm{CKI}$, and $24 \mathrm{~h}$ for further study.

\section{Wound healing assay}

T24 cells were respectively seeded into 6-well plates at a density of $2 \times 10^{5}$ cells/well. A sterile pipette tip was used to scratch cell wounds and phosphate-buffered saline was used to wash off the scraped cells. Next, different concentrations of CKI $(0,120$, or $160 \mu \mathrm{g} / \mathrm{mL})$ were added for further 
incubation. Finally, a microscope and a camera were used to record cell wound gaps of each group at 0,24 , and $48 \mathrm{~h}$. Areas of wounds were measured with ImageJ software (version 1.8.0, https://imagej. nih.gov/ij/).

\section{Apoptosis assay}

T24 cells were cultured with CKI $(0,120$, or 160 $\mu \mathrm{g} / \mathrm{mL}$ ) for $24 \mathrm{~h}$. Cells were digested with $0.25 \%$ trypsin without ethylene diamine tetraacetic acid and centrifuged at $200 \times \mathrm{g}$ for $1 \mathrm{~min}$. Cells were collected and the density in each group was adjusted to $1 \times 10^{6}$ cells $/ \mathrm{mL}$. Subsequently, $5 \mu \mathrm{L}$ of Annexin V-FITC and $5 \mu \mathrm{L}$ of propidium iodide were added to each group. After incubation for 15 min at room temperature in the dark, cell apoptosis was detected by flow cytometry (BD Biosciences, Franklin Lakes, NJ) within $1 \mathrm{~h}$.

\section{Quantitative RT-qPCR analysis}

Total RNA of T24 cells was extracted by TRIzol reagent (Invitrogen, Carlsbad, CA). Reverse transcription was performed according to kit instructions (TIANGEN, Beijing, China). Using cDNA as the amplification template, a SYBR Green I chimeric fluorescence-based method was used for real-time PCR detection in a $25 \mu \mathrm{L}$ reaction system with a PCR amplifier (Bio-Rad, Hercules, CA). Primer 5 software (Premier Biosoft International, Palo Alto, CA) was used to design corresponding primer sequences (Additional file 1: Table 1). Primers were synthesized by Shanghai General Biotech (Shanghai, China). $\beta$-actin was used as the reference gene to calculate relative expression of target genes as $2^{-\triangle \triangle \mathrm{CT}}$ values.

\section{Automatic western blotting}

T24 cells were seeded in 6-well plates at a density of $2 \times 10^{5}$ cells/well. After treatment with CKI (0, 120 , or $160 \mu \mathrm{g} / \mathrm{mL}$ ) and incubation for $24 \mathrm{~h}$, total cell protein of T24 cells was lysed and extracted by radioimmunoprecipitation assay buffer (Sigma, St. Louis, MO) containing $1 \%$ protease inhibitor. Next, the protein concentration of each group was detected with a bicinchoninic acid detection kit (Solarbio, Beijing, China). Sample proteins were separated by electrophoresis and transferred to polyvinylidene fluoride membranes by wet transfer membrane method. Membranes were blocked with Rapid Blocking Buffer (Beyotime, Shanghai, China) for $10 \mathrm{~min}$ and then incubated with primary antibodies at $4{ }^{\circ} \mathrm{C}$ overnight. Next, membranes were cultured with a horseradish peroxidase-conjugated anti-rabbit secondary antibody at room temperature. After 2-h incubation, enhanced chemiluminescence solution membranes were used to develop the target protein for $2 \mathrm{~min}$. Protein blots were exposed with an automatic chemiluminescence image analysis system (Tanon 4200, Shanghai, China). The results were analyzed using ImageJ software.

\section{Statistical analysis}

Statistical analyses were performed using GraphPad Prism 5.0 software (GraphPad Software, La Jolla, $\mathrm{CA})$. The results are shown as mean \pm standard deviation. A t-test was adopted for comparisons between two groups, while one-way analysis of variance was used to compare means of multiple groups. Statistical analyses were performed using SPSS 25.0 software (IBM Corp., Armonk, NY). P < 0.05 was considered indicative of a statistically significant difference.

\section{Results}

\section{CKI active components and targets}

In total, 113 ingredients of Sophora flavescens Aiton were selected from the TCMSP database, and 53 active components of Heterosmilax yunnanensis Gagnep were obtained through literature review. Using Lipinski's rule, 26 and 9 active components were identified in the Sophora flavescens Aiton and Heterosmilax yunnanensis Gagnep, respectively. Therefore, 35 compounds were regarded as active compounds of CKI for our further research (Additional file 2: Table 2). Targets of these active compounds were obtained from TCMSP and SwissTargetPrediction. After removing overlapped targets, a total of 268 
targets of CKI remained.

\section{Differential gene expression results of bladder cancer}

Raw data from six independent datasets (GSE100926, GSE65635, GSE61615, GSE52519, GSE27448, and GSE7476) were downloaded from the GEO database. DEGs were analyzed with $\mathrm{R}$. Detailed information of the filtered GEO datasets is summarized in Additional file 3: Table 3. DEGs are represented in the volcano plots in Fig. 2 as significantly upregulated (red) or downregulated (green) genes. After removing overlapped genes, a total of 5500 DEGs from the six bladder cancer datasets were obtained.

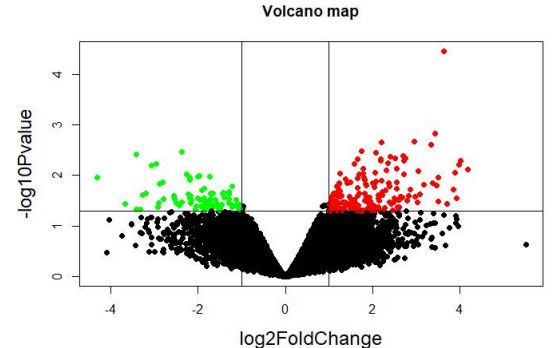

GSE100926

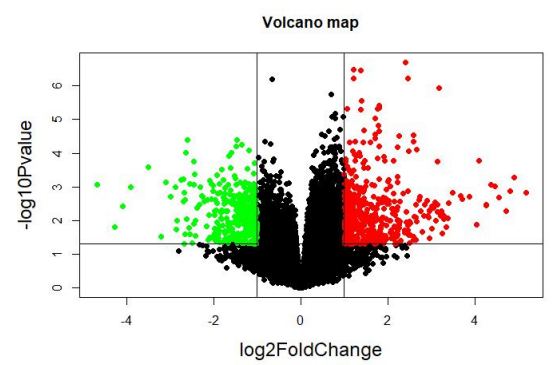

GSE52519

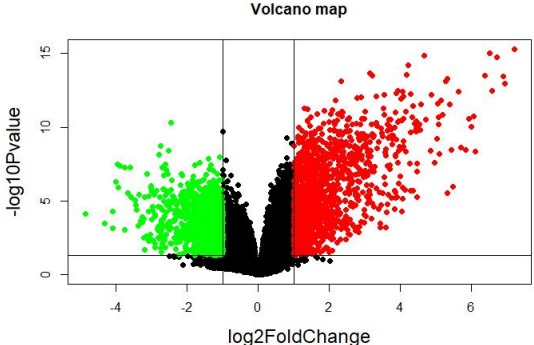

GSE65635

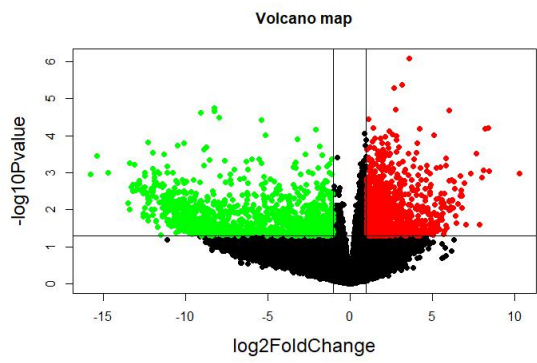

GSE27448

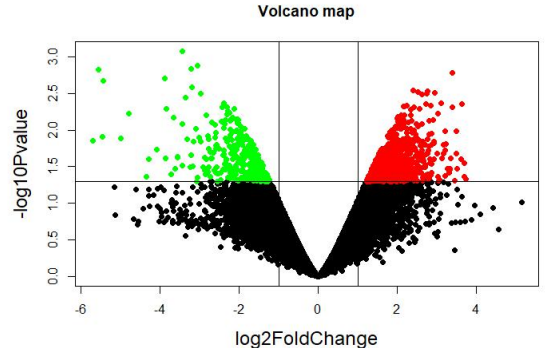

GSE61615

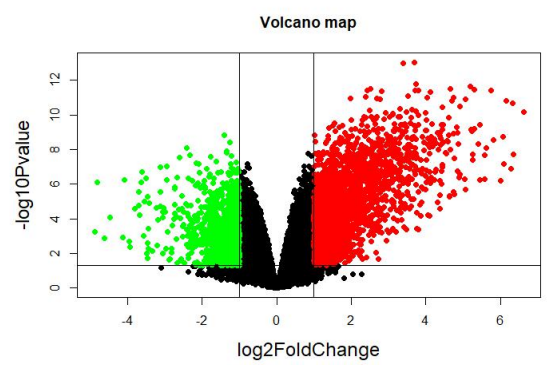

GSE7476

Fig. 2 Identification of DEGs in six GEO datasets

\section{Analysis of CKI components and bladder cancer DEGs interaction network}

The 131 intersecting genes obtained by Venn diagram are potential targets of CKI for the treatment of bladder cancer (Fig. 3a). To further clarify relationships between active compounds and potential targets, an active compounds-targetsdisease network was generated (Fig. 3b). The network contains 166 nodes and 430 edges. Blue nodes represent target genes, while peripheral nodes represent the active compound. Connections between intermediate and peripheral nodes represent interactions between the active compound and target. Furthermore, the network shows that one active target can act on different active compounds, and one compound can also act on different targets, fully reflecting the multi-component and multi-target characteristics of TCM. The obviously complex network relationship between active components and targets suggests that TCM could treat diseases in a multi-component and multi-target way. 


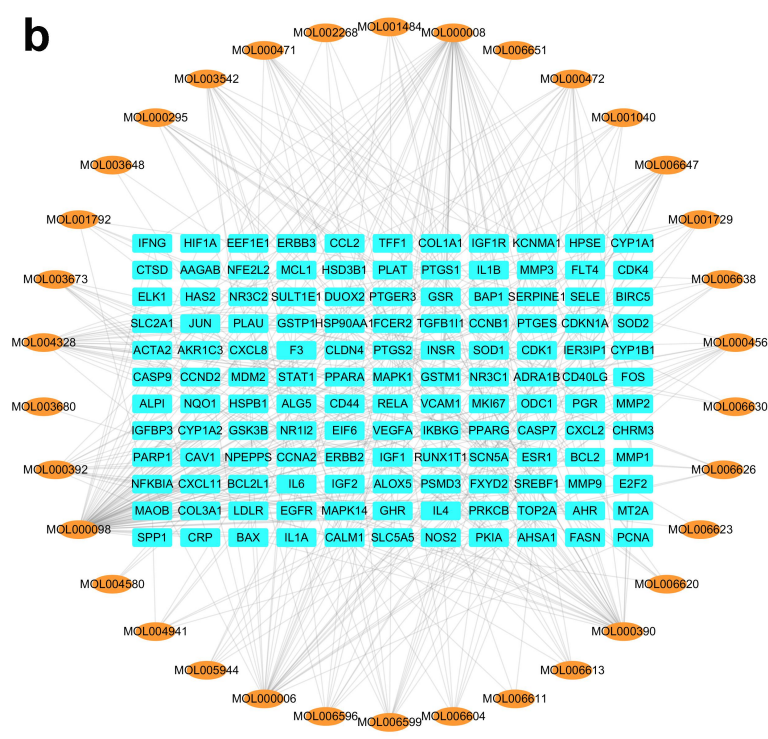

Fig. 3 a Venn diagram of overlapping genes from CKI and DEGs. b Drug-target-disease network

\section{GO and KEGG pathway enrichment analysis}

GO analyses and KEGG pathway enrichment analyses were performed using the DAVID database for a deep understanding of overlapped targets. A total of $178 \mathrm{GO}$ enrichment results and 100 KEGG signaling pathways were obtained. The top four significantly enriched GO terms, filtered by false discovery rate value, are listed (Fig. 4a). The results show that these targets were mainly involved in "apoptotic signaling pathway",

a

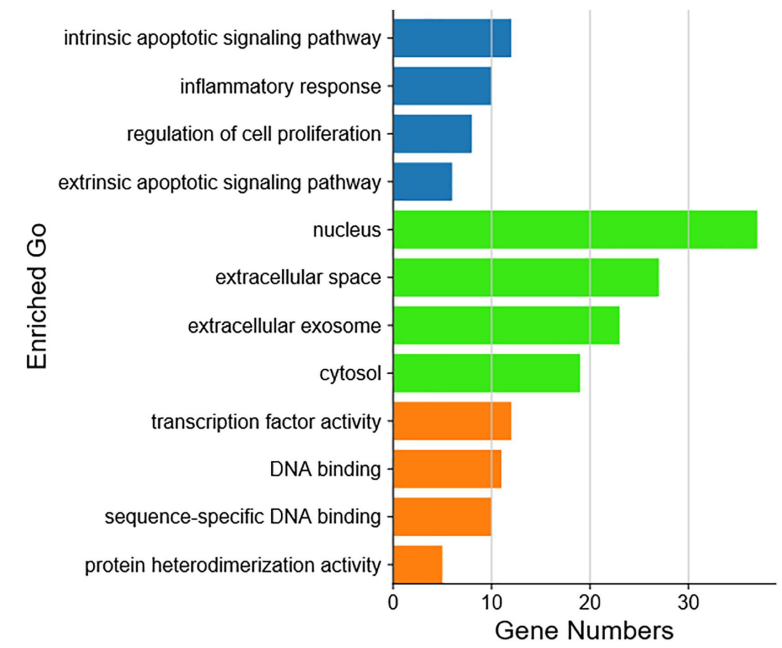

"inflammatory response", and "regulation of cell proliferation" for biological process (BP); "transcription factor activity" and "protein heterodimerization activity" for molecular function (MF); and "extracellular space", "nucleus", and "cytosol" for cellular component (CC). Additionally, six main KEGG pathways are represented, including "cancer", "bladder cancer", "proteoglycans in cancer", "TNF signaling pathway", "PI3K-Akt signaling pathway", and "HIF-1 signaling pathway" (Fig. 4b).

b

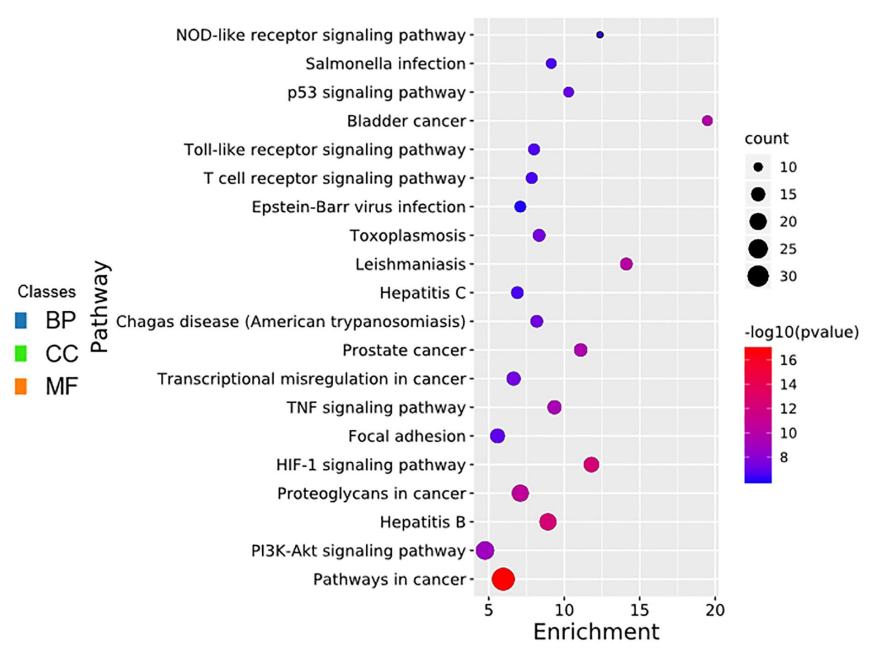

Fig. 4 a GO enrichment analysis of common targets. The top four significantly enriched terms in $\mathrm{BP}$, $\mathrm{CC}$, and $\mathrm{MF}$ categories. b The top $20 \mathrm{KEGG}$ pathways identified by enrichment analysis of putative targets 
including 34 target genes was selected as hub genes

A human PPI dataset from the STRING database of CKI for the treatment of bladder cancer (Fig. 5c). was applied (Fig. 5a) to identify hub genes using Additional file 4: Table 4 shows the basic the MCODE algorithm (Fig. 5b). A module

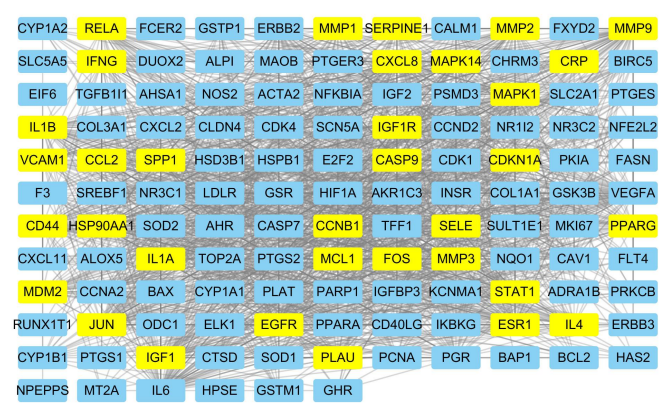

a
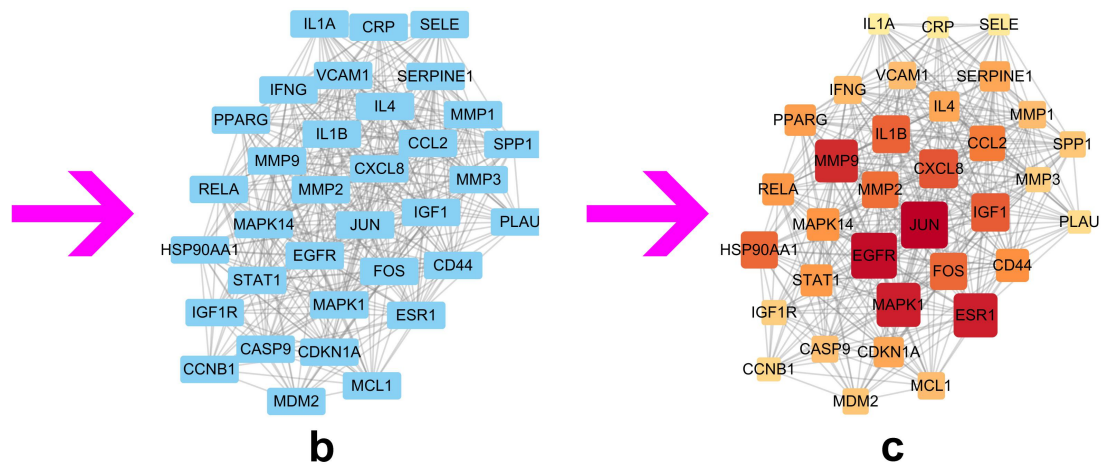

Fig. 5 PPI network, module analysis, and hub gene identification

CKI inhibited cell proliferation and migration of T24 cells in vitro

As is shown in Fig. 6a, CKI reduced T24 cell proliferation in a time- and concentration-dependent manner. The $\mathrm{IC}_{50}$ of CKI was $141.39 \pm 1.23 \mu \mathrm{g} / \mathrm{mL}$ at $24 \mathrm{~h}$. Furthermore, the effect of CKI on migration of T24 cells was observed as rates of wound healing in a cell scratch assay. The results showed that CKI significantly suppressed the migration of T24 cells compared with the control group (Fig. 6b,c).

a

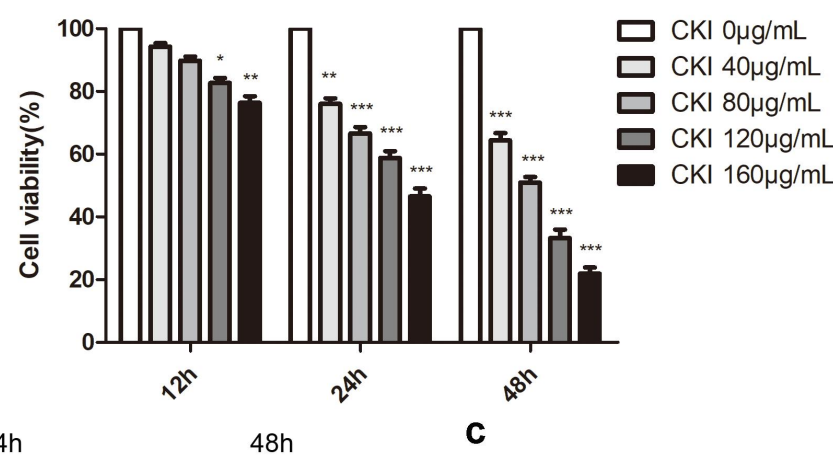

b
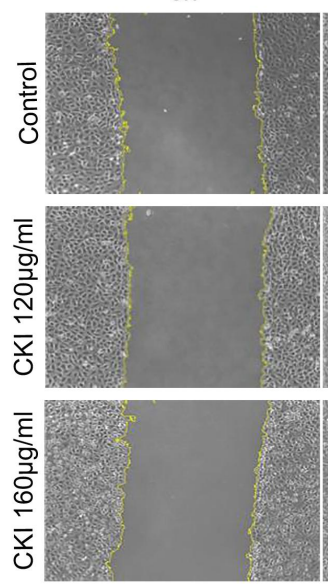

$24 \mathrm{~h}$
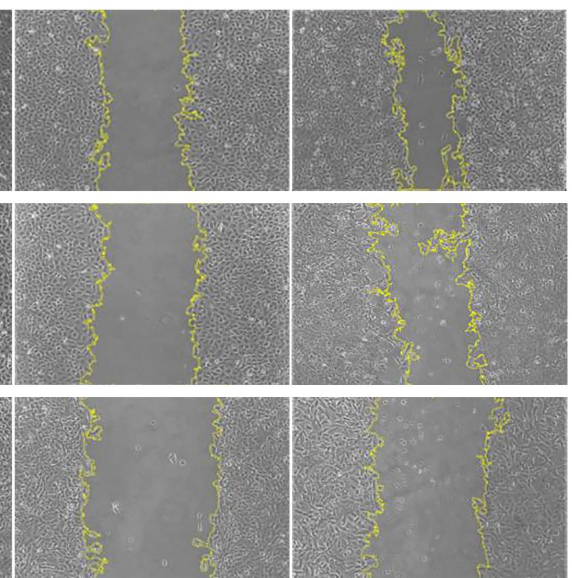

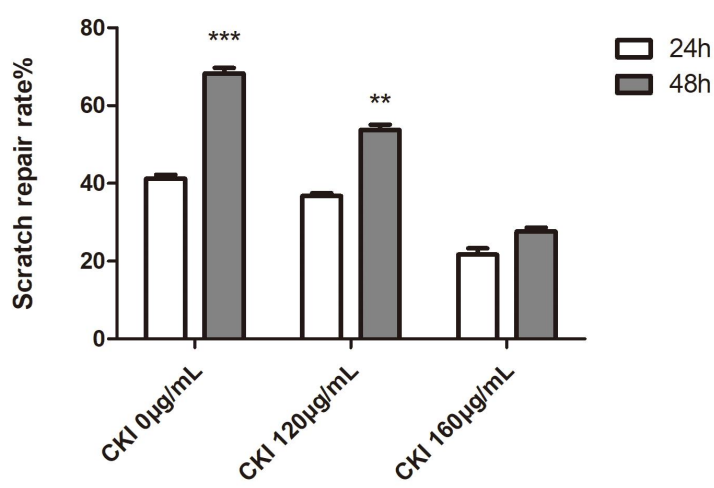


Fig. 6 CKI significantly inhibited cell proliferation and migration. a The survival rate of T24 cells after treatment with CKI for 12, 24, and $48 \mathrm{~h}$. b Representative cell wound healing pictures. c Scratch repair rate of T24 cells after culture with CKI for 24 and 48 h. $* \mathrm{P}<0.05, * * \mathrm{P}<0.01$ and $* * * \mathrm{P}<0.001$ vs. control

CKI induced apoptosis of bladder cancer T24 cells

To evaluate how CKI causes death of T24 cells, flow cytometry was used to investigate apoptosis in vitro. As presented in Fig. 7, CKI significantly
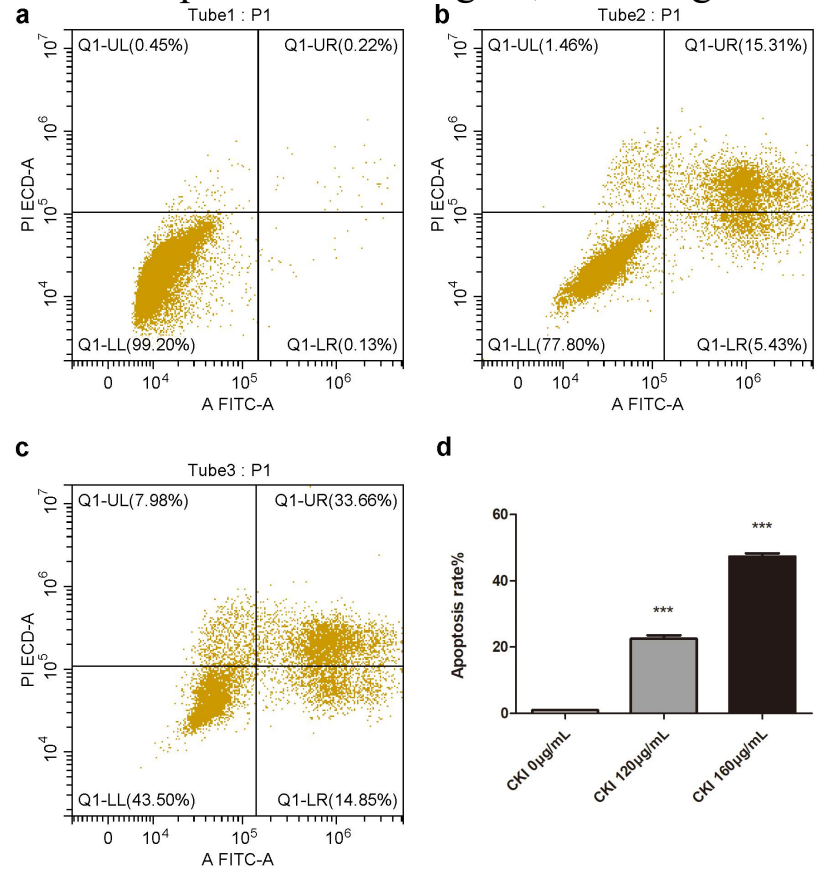

d

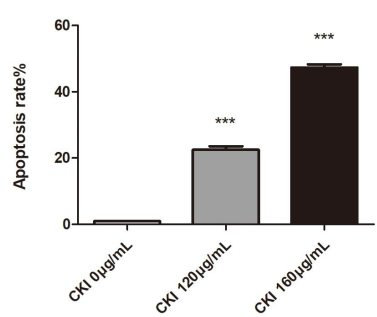

\section{RT-qPCR results}

Compared with the control group, mRNA expression of EGFR and JUN was markedly downregulated in CKI (120 and $160 \mu \mathrm{g} / \mathrm{mL})$ groups $(\mathrm{P}<0.05)$. Compared with the control group, mRNA expression levels of MMP-9 and

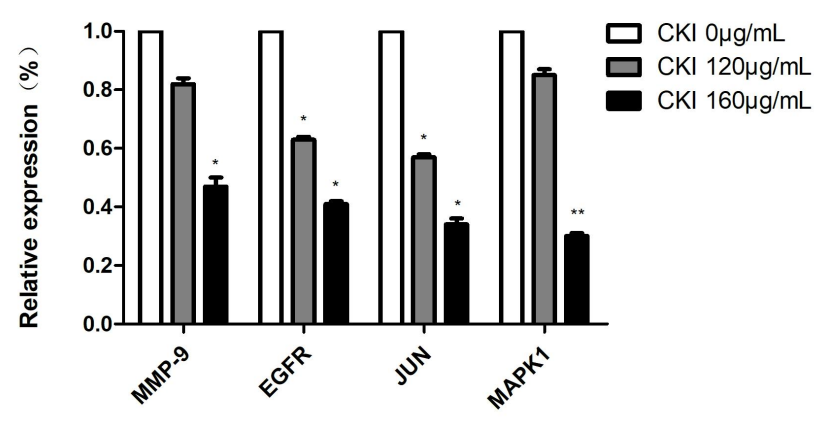

\section{Western blot results}

The crucial protein targets of CKI-treated bladder cancer predicted by network pharmacology were enhanced the apoptotic rate of $\mathrm{T} 24$ cells in a dose-dependent manner. Apoptotic rates in each group were $0.93 \pm 0.03 \%(0 \mu \mathrm{g} / \mathrm{mL}), 22.50 \pm 0.82 \%$ $(120 \mu \mathrm{g} / \mathrm{mL})$, and $47.34 \pm 0.75 \%(160 \mu \mathrm{g} / \mathrm{mL})$. There were significant differences in each group of data $(\mathrm{P}<0.001)$.

Fig. 7 Flow cytometry was used to detect the apoptosis of T24 cells after treatment with CKI at concentrations of 0 , 120 , and $160 \mu \mathrm{g} / \mathrm{mL}$ for $24 \mathrm{~h}$ a, b, c. d Apoptotic rates increased in a dose-dependent manner. $* * * \mathrm{P}<0.001$ vs. control

mitogen-activated protein kinase 1 (MAPK1) were downregulated in CKI groups, but these differences were only significant in the group treated with 160 $\mu \mathrm{g} / \mathrm{mL}$ CKI $(\mathrm{P}<0.05)$. These results indicated that after treatment with CKI, expression of MMP-9, EGFR, JUN and MAPK1 in T24 cells was downregulated (Fig. 8).

Fig. 8 RT-qPCR of MMP-9, EGFR, JUN, and MAPK1 mRNA expression in T24 cells after treatment with CKI for 24 h. $* \mathrm{P}<0.05,{ }^{*} \mathrm{P}<0.01$ vs. control

validated by western blot. As is shown in Fig. 9, CKI significantly inhibited protein expression of JUN, EGFR, MAPK1, and MMP-9 in T24 cells. 

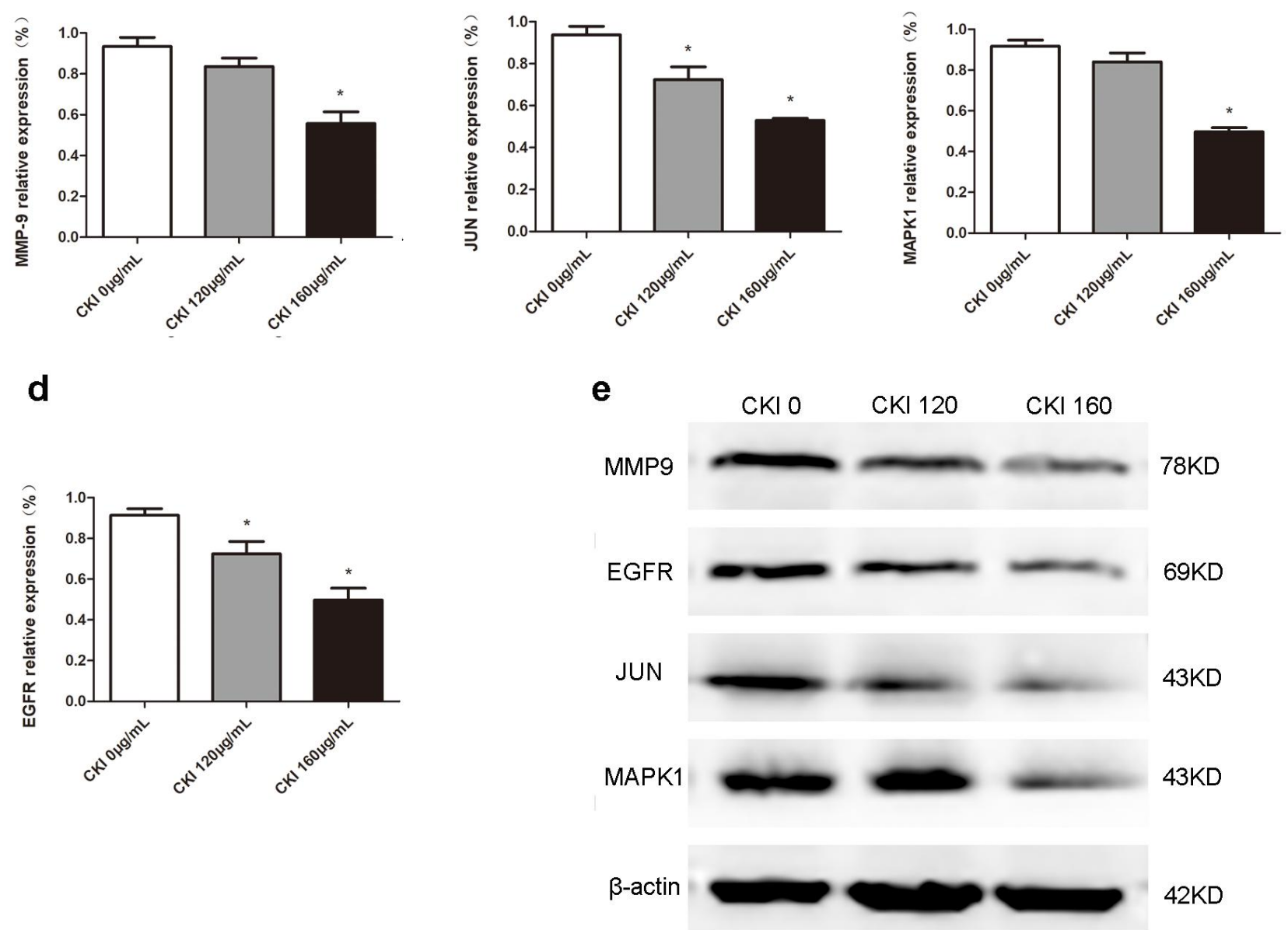

Fig. 9 CKI can alter key protein expression levels in T24 cells. $\beta$-actin was used as an internal control. Protein expression levels of MMP-9 a, EGFR b, JUN $\mathbf{c}$, and MAPK1 d are visualized by statistical diagrams. e Representative images of protein bands. $* \mathrm{P}<0.05$ vs. control

\section{Discussion}

In recent years, many medicinal plants have been shown to have functions for the treatment of various diseases. CKI is a TCM preparation prepared by processing and extracting two Chinese herbs, Sophora flavescens Aiton and Heterosmilax yunnanensis Gagnep. Research showed that 20-200 $\mu \mathrm{g} / \mathrm{mL}$ Radix Sophorae Flavescentis could induce apoptosis of human gastric cancer cells by downregulating Bcl-2 and upregulating Bax [29]. In addition, a network pharmacology method predicted that CKI inhibited the growth and metastasis of gastric cancer cells by regulating vital targets and important related pathways [30]. Previous research confirmed that five compounds (sophocarpine, matrine, oxymatrine, oxysophocarpine, and xanthohumol) of Radix
Sophorae Flavescentis exerted anti-prostate cancer effects by affecting cell proliferation and apoptosis [31]. Moreover, the effect of CKI was validated to perturb three pathways (cell cycle, energy metabolism, and DNA repair) in the human breast adenocarcinoma cell line MDA-MB-231 and hepatocellular carcinoma cell line Hep G2 [32]. In addition, animal and in vitro experiments with MCF-7 cells indicated that CKI suppressed breast cancer tumor growth by downregulating the $\mathrm{Wnt} / \beta$-catenin pathway [33]. As an antioxidant, CKI inhibited proliferation of human acute myeloid leukemia by regulating the Prdxs/ROS/Trx1 signaling pathway, suggesting its promise as potential treatment to this disease [34]. Furthermore, according to combined quantitative analysis of components, CKI exerts anti-hepatocellular carcinoma effects by inhibiting the proliferation and 
migration of SMMC-7721 cells [11]. The immunologic function of patients with locally advanced non-small cell lung cancer who receive concomitant radiochemotherapy could be remarkedly increased by the treatment of CKI in clinical research [35]. Additionally, as an adjunctive therapy for the treatment of non-small-cell lung cancer, CKI improved clinical efficiency, Karnofsky Performance Status, and immune function, and reduced adverse reactions compared with chemotherapy alone [55]. A systematic review and meta-analysis showed that CKI could improve the total pain relief rate and enhance the quality of life of patients with bone cancer [36]. Moreover, an in vitro cell experiment and transcriptome analysis showed that CKI enhanced the cytotoxic effects of doxorubicin on human epidermoid squamous carcinoma A431 cells [37]. Collectively, the reports described above demonstrated that CKI can exert anti-cancer effects through multiple pathways.

Bladder cancer is one of the most common urological cancers. In 2020, approximately 81,400 new bladder cancer cases and 17,980 deaths were reported in the United States [38]. However, even though bladder cancer is common, it is not well treated by current therapeutic strategies, and recurrence rates of both NMIBC and MIBC after surgical and chemotherapy management remain high [39]. Postoperative recurrence and cancer cell metastasis are the main causes of death of patients with bladder cancer [40]. The development of bladder cancer involves many signaling pathways and types of intracellular signaling molecules [41]. Epidermal growth factor receptor (EGFR) plays an important role in tumor angiogenesis, and its expression is correlated with the grade and stage of bladder cancer [42]. Triggering of EGFR signals by vascular endothelial cells that interact with bladder cancer cells and tissues can promote tumor progression [43]. Furthermore, with regard to the treatment of bladder cancer, downregulation of EGFR can inhibit the migration of cancer cells [44]. Matrix metalloproteinases (MMPs) can promote malignant tumor cell proliferation and support the tumor microenvironment [45]. In addition, MMP-9 is used as a marker gene for early diagnosis and prognosis of bladder cancer [46]. Other studies found that regulating the SP1/MMP9 pathway can suppress the migration of bladder cancer, whereas tumor progression was promoted by upregulation of MMP9 in bladder cancer [47, 48]. The transcription factor Jun proto-oncogene (JUN) exhibits differential expression and strong activity in various cancers [49]. Significantly higher expression of JUN was observed in invasive bladder cancer tissues and cell lines [50]. Moreover, regulation of the JNK/c-Jun signaling pathway can increase the sensitivity of bladder cancer cells to chemotherapy drugs [51]. The MAPK signaling pathway also plays an important role in the occurrence and development of bladder cancer [52]. Indeed, the activation status of the MAPK pathway can be used as a prognosticator for oncologic outcomes and targeted therapy of advanced bladder cancer [53]. MAPK1, a member of the MAPK family, is involved in processes such as cell proliferation, differentiation, and transcriptional regulation. Silencing of MAPK1 can inhibit the proliferation and migration of bladder cancer T24 cells [54]. To summarize, by regulating related genes and signaling pathways, CKI can influence the occurrence and development of bladder cancer.

TCM theory posits that bladder cancer is associated with hematuric strangury and retention of urine. Its pathogenesis results from the accumulation of damp-heat and blood stasis, long-term deficiency of the spleen and kidney, and dysfunction of qi in transformation. A meta-analysis showed that the use of TCM as adjuvant therapy for bladder cancer chemotherapy could significantly reduce recurrence rates, as well as the toxicity and side effects caused by chemotherapy, thus improving the quality of life and prolonging the survival of patients [55].

In CKI, Sophora flavescens Aiton has the effect of clearing heat and expelling dampness and diuresis, while Heterosmilax yunnanensis Gagnep can clear damp and invigorate the spleen and stomach. The efficacy of these two drugs is very suitable for the pathogenesis of bladder cancer. CKI is used for adjuvant therapy of bladder cancer 
chemotherapy and radiotherapy, and has achieved good clinical efficacy. Research indicates that CKI can inhibit the invasion and metastasis of bladder tumors by reducing vascular endothelial growth factor expression [56]. To clarify the molecular mechanism of CKI in treatment of bladder cancer, we further explore related pathways and key targets using network pharmacology and bioinformatic methods. The 35 main active compounds of CKI related to the treatment of bladder cancer, such as sophocarpine, sophoridine, formononetin, cis-dihydroquercetin, matrine, luteolin, glyceollin, kurarinol, kushenin, pterocarpine, apigenin, quercetin, daidzein, and emodin, have proven anti-cancer effects; however, the anti-bladder cancer mechanism of CKI should integrate the anti-cancer effects of all these active ingredients. Therefore, we examined 131 potential targets of CKI in treatment of bladder cancer for follow-up exploration. In the present study, we first screened DEGs from six GEO datasets for bladder cancer, and discovered that cell apoptosis and regulation of cell proliferation may be the key biological processes involved in bladder cancer. Moreover, tumor necrosis factor (TNF) and phosphoinositide 3 kinase (PI3K)-Akt signaling pathways were identified as potentially important KEGG pathways related to the effect of CKI for treatment of bladder cancer. A PPI network was constructed using the STRING database, and hub genes were selected using MOCDE in Cytoscape software. Next, we conducted experimental verification of important biological processes and hub genes. We found that CKI significantly inhibited the proliferation and migration of bladder cancer T24 cells, and induced their apoptosis. Moreover, significantly downregulated expression of important genes and proteins, such as MMP-9, EGFR, JUN and MAPK1, was observed.

Scientific research that can be applied to existing clinical treatments is meaningful for the field of medicine. The concentration of CKI used in this study was the optimal concentration verified by pre-experiments, and within the range of blood drug concentrations produced by the human body following administration of a clinical dose.
Therefore, the research results have certain clinical application and reference value. Further investigations are still necessary to unravel the mechanism by which CKI is effective for the treatment of bladder cancer. We have much to learn and will continue the quest to discover more effective therapies [57].

\section{Conclusions}

In conclusion, according to network pharmacology and bioinformatics predictions, we conducted cell experiments in vitro to validate the underlying mechanisms by which CKI effectively treats bladder cancer. We elucidated that CKI could significantly inhibit bladder cancer T24 cell proliferation and migration, and induce apoptosis. Potential signaling pathways involved EGFR, PI3K/Akt, and MAPK via inhibition of EGFR, JUN, MMP-9, and MAPK1 hub gene expression. Overall, our findings suggest that CKI can treat bladder cancer through multiple targets and signaling pathways, thus providing deeper pharmacological mechanisms for future clinical research.

\section{Additional files}

Additional file 1: Table 1. Primer sequences.

Additional file 2: Table 2. Active components of CKI.

Additional file 3: Table 3. GEO data information. Additional file 4: Table 4. Basic information of hub genes.

\section{Abbreviations}

CKI (Compound Kushen Injection); DAVID (Database Visualization and Integrated Discovery); DEGs (differentially expressed genes); Kyoto Encyclopedia of Genes and Genomes (KEGG); non-muscle-invasive disease (NMIBC); muscle-invasive bladder cancer (MIBC); Traditional Chinese medicine (TCM); gene expression omnibus (GEO); Traditional Chinese Medicine Systems Pharmacology (TCMSP); drug likelihood (DL); hydrogen bond acceptors (Hacc); hydrogen bond donors (Hdon); octanol-water 
partition coefficient lesser than five $(\log \mathrm{P})$; Gene Ontology (GO); protein-protein interaction (PPI); molecular complex detection (MCODE); biological process (BP); cellular component (CC); molecular function (MF); Epidermal Growth Factor Receptor (EGFR); Matrix metallopeptidase 9 (MMP-9); mitogen-activated protein kinase 1 (MAPK1); Jun Proto-Oncogene (JUN).

\section{Acknowledgments}

Not applicable.

\section{Funding}

This research was supported by the National Natural Science Foundation of Hebei (No. H2018201179), Health and Family Planning Commission of Hebei (No. 20190948), Hebei Universities Research Project of Science and Technology (No. QN2019146), National Undergraduate Innovation and Entrepreneurship Training Program Project (No. 2020376), Medical Science Foundation of Hebei University (No.2020A15) and Hebei University Medical Discipline Cultivation Project. We thank Liwen Bianji, Edanz Group China (www.liwenbianji.cn/ac), for editing the English text of a draft of this manuscript.

\section{Authors' Contributions}

GW Zhang and LH Zhang designed the research. LH Zhang, WY Zhang, and JM Xiong performed the experiments. XM Duan, LN Hai, YL Zhang, MM Zhang, and GF Qin were involved in data collection. LH Zhang analyzed the experimental data and wrote the manuscript, and GW Zhang revised the manuscript. All the authors have read and approved the final manuscript.

\section{Availability of data and materials}

The datasets used and/or analyzed during the current study are available from the corresponding author on reasonable request.

\section{Ethics approval and consent to participate}

Not applicable.

\section{Consent for publication}

Not applicable.

\section{Competing interests}

The authors declare that they have no competing interests.

\section{Author details}

${ }^{1}$ College of Traditional Chinese Medicine, Hebei University, Baoding, Hebei, 071002, China. ${ }^{2}$ Beijing Zhendong Guangming Pharmaceutical Research Institute Limited Company, Beijing, 100085, China. ${ }^{3}$ Academy for Advanced Interdisciplinary Studies, Peking University, Beijing, 100871, China.

\section{References}

1. Bray F, Ferlay J, Soerjomataram I, et al. Global cancer statistics 2018: GLOBOCAN estimates of influence and mortality worldwide for 36 cancers. CA: A Cancer Journal for Clinicians. 2018;68(6):394-424. https://doi.org/10.3322/caac.21492

2. Kates M, Date A, Yoshida T, et al. Preclinical Evaluation of Intravesical Cisplatin Nanoparticles for Non-Muscle-Invasive Bladder Cancer. Clin Cancer Res. 2017;23(21):6592-601. https://doi.org/10.1158/1078-0432.CCR-17-1082

3. Letašiová S, Medve'ová A, Šovčíková A, et al. Bladder cancer, a review of the environmental risk factors. Environmental Health. 2012;11 Suppl 1(Suppl 1):S11. https://doi.org/10.1186/1476-069X-11-S1-S11

4. Christof Seidl. Targets for Therapy of Bladder Cancer. Seminars in Nuclear Medicine. 2020;50(2):162-170. https://doi.org/10.1053/j.semnuclmed.2020.02.006

5. Vastrad B, Vastrad C. Screening and identification of potential prognostic biomarkers in bladder urothelial carcinoma: Evidence from bioinformatics analysis. Gene Reports. 2020;20. https://doi.org/10.1016/j.genrep.2020.100658 
6. Bauer W, Lamche M, Paul Schramek. Interdisciplinary strategies for the treatment of bladder cancer. Wiener Medizinische Wochenschrift. 2007;157(7-8):153-156. https://doi.org/10.1007/s10354-007-0399-3

7. Tang H, Shu P, Liu S, et al. Traditional Chinese Medicine in Oncotherapy: The Research Status. Nutrition and Cancer. 2020;72(6):992-998. https://doi.org/10.1080/01635581.2019.1664599

8. Ding A, Patel J, Auyeung V. Understanding the Traditional Chinese Medicine (TCM) consultation: Why do patients adhere to treatment? Complementary Therapies in Clinical Practice. 2020;39. https://doi.org/10.1016/j.ctcp.2020.101139

9. Wu L, Wang G, Liu S, et al. Synthesis and biological evaluation of matrine derivatives containing benzo- $\alpha$-pyrone structure as potent anti-lung cancer agents. Scientific Reports. 2016;6:35918. https://doi.org/10.1038/srep35918

10. Wang W, You R, Qin W, et al. Anti-tumor activities of active ingredients in Compound Kushen Injection. Acta Pharmacologica Sinica. 2015;36(6):676-679. https://doi.org/10.1038/aps.2015.24

11. Gao L, Wang K, Zhou Y, et al. Uncovering the anticancer mechanism of Compound Kushen Injection against $\mathrm{HCC}$ by integrating quantitative analysis, network analysis and experimental validation. Scientific Reports. 2018;8,624. https://doi.org/10.1038/s41598-017-18325-7

12. Wang X, Liu J, Lin H, et al. A multicenter randomized controlled open-label trial to assess the efficacy of compound kushen injection in combination with single-agent chemotherapy in treatment of elderly patients with advanced non-small cell lung cancer: study protocol for a randomized controlled trial. BioMed Central. 2016;17(1):124. https://doi.org/10.1186/s13063-016-1231-6

13. Aung T. N, Nourmohammadi S, Qu Z, et al. Fractional Deletion of Compound Kushen Injection Indicates Cytokine Signaling Pathways are Critical for its Perturbation of the Cell Cycle. Scientific Reports. 2019;9(1). https://doi.org/10.1038/s41598-019-50271-4

14. He R, Ou S, Chen S, et al. Network Pharmacology-Based Study on the Molecular Biological Mechanism of Action for Compound Kushen Injection in Anti-Cancer Effect. Medical science monitor : international medical journal of experimental and clinical research. 2020;26: e918520. https://doi.org/10.12659/MSM.918520

15. Luo Y, Feng Y, Song L, et al. A network pharmacology-based study on the anti-hepatoma effect of Radix Salviae Miltiorrhizae. Chinese medicine. 2019;14:27. https://doi.org/10.1186/s13020-019-0249-6

16. Zhang Z, Yi P, Yang J, et al. Integrated network pharmacology analysis and serum metabolomics to reveal the cognitive improvement effect of Bushen Tiansui formula on Alzheimer's disease. Journal of Ethnopharmacology. 2019;249:112371. https://doi.org/10.1016/j.jep.2019.112371

17. Wang J, Chi S, Huang Z, et al. Comprehensive characterization of differentially expressed genes in thyroid cancer. Future oncology. 2017;13(24). https://doi.org/10.2217/fon-2017-0168

18. Ru J, Li P, Wang J, et al. TCMSP: a database of systems pharmacology for drug discovery from herbal medicines. Journal of Cheminformatics. 2014;6(1):13. https://doi.org/10.1186/1758-2946-6-13

19. Li S, Wang N, Hong M, et al. Hepatoprotective Effects of a Functional Formula of Three Chinese Medicinal Herbs: Experimental Evidence and Network Pharmacology-Based Identification of Mechanism of Action and Potential Bioactive Components. Molecules. 2018;23(2):352. https://doi.org/10.3390/molecules23020352

20. Wang S, Tong Y, Ng T. et al. Network pharmacological identification of active compounds and potential actions of Erxian decoction in alleviating menopause-related symptoms. Chin Med. 2015;10,19. https://doi.org/10.1186/s13020-015-0051-z

21. Antoine D, Olivier M, Vincent Z.

Swiss TargetPrediction: updated data and new features for efficient prediction of protein targets of small molecules. Nucleic Acids Research. 2019;W1:47. https://doi.org/10.1093/nar/gkz382

22. Ritchie ME, Phipson B, Wu D, et al. limma powers differential expression analyses for RNA-sequencing and microarray studies. Nucleic Acids Res.

2015;43,e47. https://doi.org/10.1093/nar/gkv007

23. Yeung N, Cline M, Kuchinsky A, et al. Exploring Biological Networks with Cytoscape Software. Current Protocols in Bioinformatics. 2008;23(1). https://doi.org/10.1002/0471250953.bi0813s23.

24. Huang DW, Sherman BT, Lempicki RA. Systematic 
and integrative analysis of large gene lists using DAVID bioinformatics resources. Nature Protocols:

Recipes for Researchers. 2009;4(1).

https://doi.org/10.1038/nprot.2008.211

25. Ashburner M., Ball CA., Blake JA., et al. Gene

Ontology: tool for the unification of biology. Nature

Genetics. 2000;25(1):25-9.

https://doi.org/10.1038/75556

26. Minoru K, Miho F, Mao T, et al. KEGG: new perspectives on genomes, pathways, diseases and drugs. Nucleic Acids Research.

2017;45(D1):D353-D361.

https://doi.org/10.1093/nar/gkw1092

27. Ashraf G M, Vasileios T, Charalampos V, et al. Protein-Protein Interaction (PPI) Network: Recent Advances in Drug Discovery. Current Drug Metabolism. 2017;18(1):5-10. https://doi.org/10.2174/138920021801170119204832

28. Han L, Qi Z, Wen JW, et al. The potential drug for treatment in pancreatic adenocarcinoma: a bioinformatical study based on distinct drug databases. Chinese Medicine. 2020;15(12):764-775. https://doi.org/10.1186/s13020-020-00309-x

29. Sun K.J, Jae S.S, Nam K.J, et al. Radix Sophorae Flavescentis inhibits proliferation and induces apoptosis of AGS human gastric cancer cells. Molecular medicine reports. 2018;19(3):1911-1918. https://doi.org/10.3892/mmr.2018.9776

30. Zhou W, Wu J, Zhu Y, et al. Study on the mechanisms of compound Kushen injection for the treatment of gastric cancer based on network pharmacology. BMC Complementary Med Ther. 2020;20, 6. https://doi.org/10.1186/s12906-019-2787-y

31. Wang Q, Xu J, Li X, et al. Comprehensive two-dimensional PC-3 prostate cancer cell membrane chromatography for screening anti-tumor components from Radix Sophorae flavescentis. Journal of Separation Science. 2017;40(13):2688-2693. https://doi.org/10.1002/jssc.201700208

32. Cui J, Qu Z, Harata-Lee Y, et al. Cell cycle, energy metabolism and DNA repair pathways in cancer cells are suppressed by Compound Kushen Injection. BioMed Central. 2019;19(1):103. https://doi.org/10.1186/s12885-018-5230-8

33. $\mathrm{Xu}$ W, Lin $\mathrm{H}$, Zhang Y, et al. Compound Kushen Injection suppresses human breast cancer stem-like cells by down-regulating the canonical
Wnt/ $\beta$-cateninpathway. J Exp Clin Cancer Res.

2011;30,103.

https://doi.org/10.1186/1756-9966-30-103

34. Jin Y, Yang Q, Liang L, et al. Compound kushen injection suppresses human acute myeloid leukaemia by regulating the Prdxs/ROS/Trx1 signalling pathway. BioMed Central. 2018;37(1):277. https://doi.org/10.1186/s13046-018-0948-3

35. Zhao Z, Liao H, Ju Y. Effect of compound Kushen injection on T-cell subgroups and natural killer cells in patients with locally advanced non-small-cell lung cancer treated with concomitant radiochemotherapy. Journal of Traditional Chinese Medicine. 2016;36(1):14-18. https://doi.org/10.1016/S0254-6272(16)30002-4

36. Yanju B, Yang L, Hua B, et al. A systematic review and meta-analysis on the use of traditional Chinese medicine compound kushen injection for bone cancer pain. Support Care Cancer 22. 2014;825-836. https://doi.org/10.1007/s00520-013-2063-5

37. Shen H, Qu Z, Harata-Lee Y, et al. A New Strategy for Identifying Mechanisms of Drug-drug Interaction Using Transcriptome Analysis: Compound Kushen Injection as a Proof of Principle. Sci Rep. 2019;9,15889. https://doi.org/10.1038/s41598-019-52375-3

38. Siegel Rebecca L, Miller Kimberly D, Jemal Ahmedin. Cancer statistics. CA: a cancer journal for clinicians. 2020;70(1):7-30. https://doi.org/10.3322/caac.21590

39. Ashish M K, Noah M H, Jason A E, et al. Bladder cancer. The Lancet. 2016;388(10061):2796-2810. https://doi.org/10.1016/S0140-6736(16)30512-8

40. Whitmore W F. Toward the Rational Management of Bladder Cancer: An Overview. Urology.1988;31(2 Suppl):5-8. https://doi.org/10.1016/S0022-5347(17)41857-X

41. Calderaro J, Rebouissou S, Koning L. PI3K/AKT pathway activation in bladder carcinogenesis. International journal of cancer.

2013;134(8):1776-1384. https://doi.org/10.1002/ijc.28518

42. Bennasroune A, Gardin A, Aunis D, et al. Tyrosine kinase receptors as attractive targets of cancer therapy. Critical Reviews in Oncology and Hematology. 2003;50(1):23-38. https://doi.org/10.1016/j.critrevonc.2003.08.004

43. Huang Z, Zhang M, Chen G, et al. Bladder cancer cells 
interact with vascular endothelial cells triggering EGFR signals to promote tumor progression.

International journal of oncology.

2019;54(5):1555-1556.

https://doi.org/10.3892/ijo.2019.4729

44. Li J, Wang F, Gao H, et al. ALDOLASE A regulates invasion of bladder cancer cells via E-cadherin-EGFR signaling. Journal of Cellular Biochemistry.

2019;120(8):13694-13705.

https://doi.org/10.1002/jcb.28642

45. Matsunaga Y, Koda M, Murawaki Y. Expression of matrix metalloproiteinases (MMPs) and tissue inhibitors of metalloproteinases (TIMPs) in hepatocellular carcinoma tissue, compared with the surrounding non-tumor tissue. Research Communications in Molecular Pathology \& Pharmacology. 2004;115-116:143-150. https://doi.org/10.1007/s00403-014-1494-2

46. Fouad H, Salem H, El-Sayed D, et al. MMP-2 and MMP-9 as prognostic markers for the early detection of urinary bladder cancer. Journal of Biochemical and Molecular Toxicology.2018;33(4):e22275. https://doi.org/10.1002/jbt.22275

47. Yan H, Li J,Ying Y, et al. MIR-300 in the imprinted DLK1-DIO3 domain suppresses the migration of bladder cancer by regulating the SP1/MMP9 pathway. Cell Cycle. 2018;17(24):2790-2801. https://doi.org/10.1080/15384101.2018.1557490

48. Qin Z, Wang Y, Tang J, et al. High LINC01605 expression predicts poor prognosis and promotes tumor progression via upregulation of MMP9 in bladder cancer. Bioscience reports. 2018;40(5). https://doi.org/10.1042/BSR20180562

49. Vogt P.K. Jun, the oncoprotein. Oncogene.

2001;20(19):2365-2377.

https://doi.org/10.1038/sj.onc. 1204443

50. Li Z, Zhu Y, Yu M, et al. c-Jun is involved in interstitial cystitis antiproliferative factor (APF)-induced growth inhibition of human bladder cancer T24 cells. Urologic Oncology: Seminars and Original
Investigations. 2013;31(2):228-233.

https://doi.org/10.1016/j.urolonc.2010.11.011

51. Liu J, Zhai R, Zhao J, et al. Programmed cell death 4 overexpression enhances sensitivity to cisplatin via the JNK/c-Jun signaling pathway in bladder cancer. International journal of oncology.

2018;52(5):1633-1642.

https://doi.org/10.3892/ijo.2018.4303

52. Chen J, Chou F, Yeh S, et al. Androgen dihydrotestosterone (DHT) promotes the bladder cancer nuclear AR-negative cell invasion via a newly identified membrane androgen receptor (mAR-SLC39A9)-mediated Gai protein/MAPK/MMP9 intracellular signaling. Oncogene. 2020;39(3):574-586. https://doi.org/10.1038/s41388-019-0964-6

53. Schulz G.B, Grimm T, Jokisch F, et al. Therapeutic and diagnostic implications of the NOTCH and MAPK pathways in urothelial bladder cancer. European Urology Supplements. 2018;17(2):e652. https://doi.org/10.1016/S1569-9056(18)31292-2

54. Wang X,Wu G, Cao G, et al. MicroRNA-335 inhibits bladder cancer cell growth and migration by targeting mitogen-activated protein kinase 1. Molecular medicine reports. 2016;14(2):1765-1770. https://doi.org/10.3892/mmr.2016.5448

55. Zhang L, Yang Y, Chen Y, et al. Traditional Chinese Medicine for Bladder Cancer: a Systematic Review and Meta-Analysis. Medical Data Mining. 2018;1(2): 68-82. https://doi.org/10.12032/mdm2018008

56. Zhang L, Sun J, Pan S, et al. Effect of Compound Kushen Injection on proliferation and invasion of bladder cancer cells. Journal of Chinese Medicinal Materials. 2017; 40(05):1208-1211. https://doi.org/10.13863/j.issn1001-4454.2017.05.047

57. Daneshmand S. Bladder Cancer: Advances and Innovations. European Urology Focus. 2020;6(4):613-614. https://doi.org/10.1016/j.euf.2020.02.012 
Figures

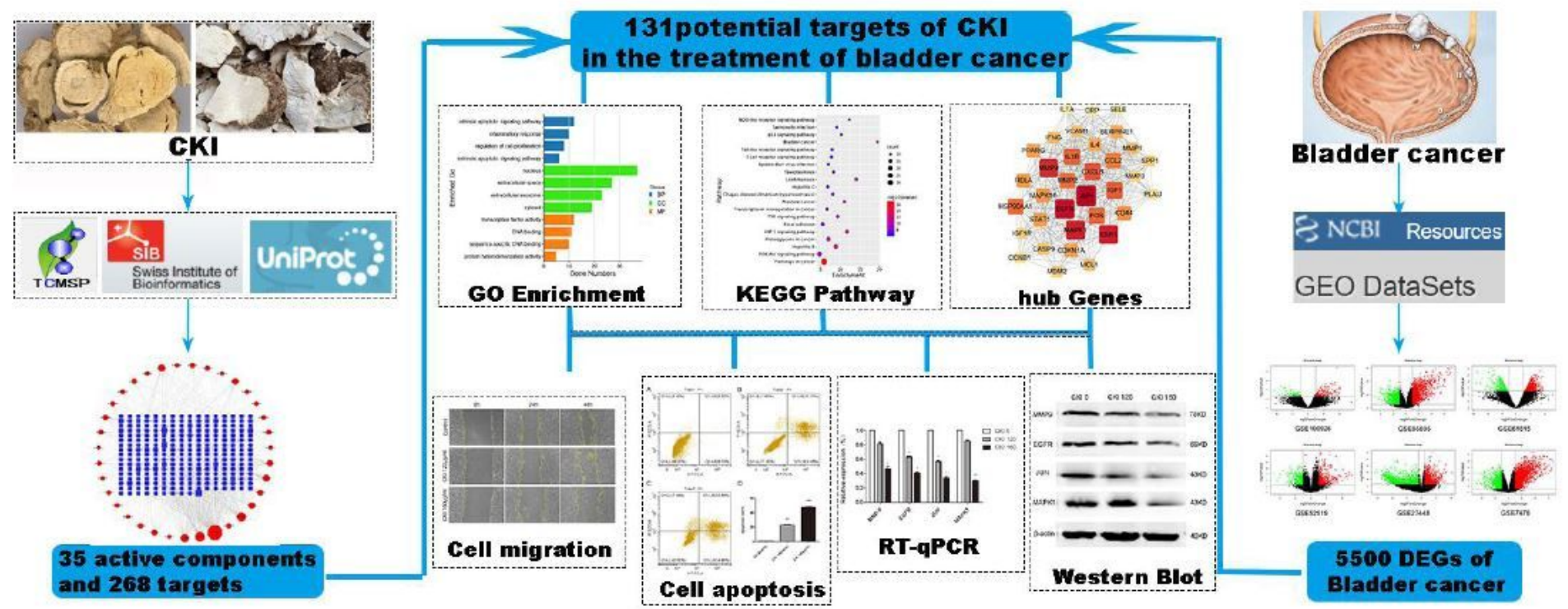

Figure 1

Scheme of the study

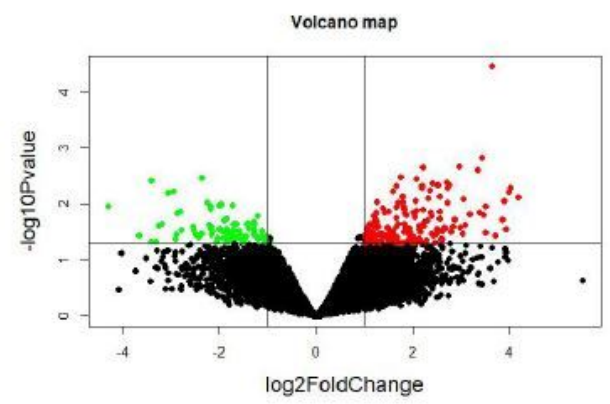

GSE100926

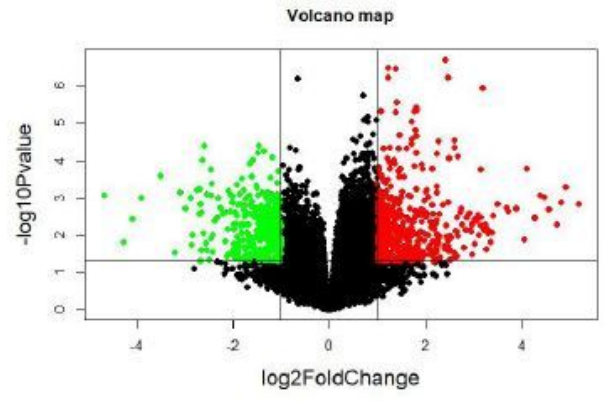

GSE52519

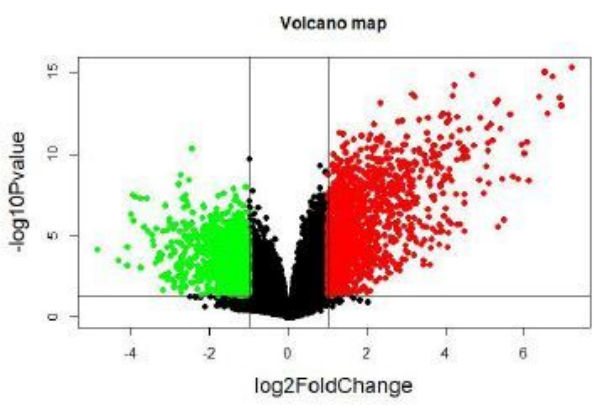

GSE65635

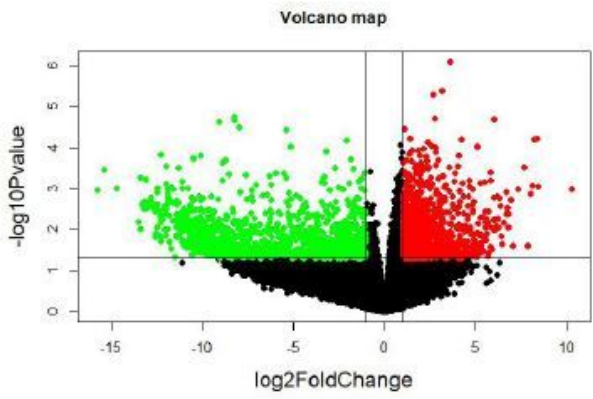

GSE27448

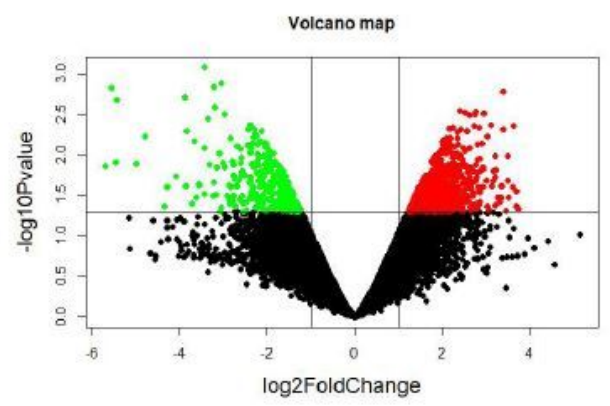

GSE61615

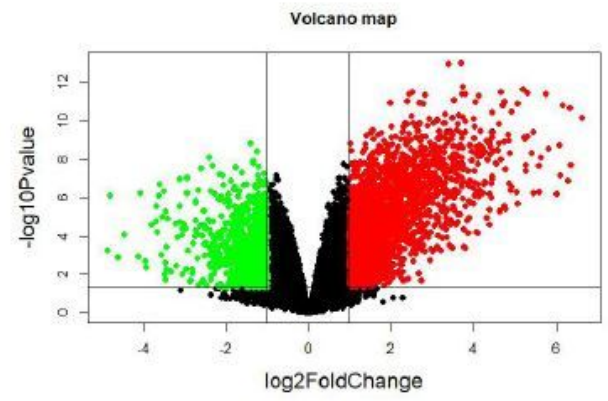

GSE7476

Figure 2

Identification of DEGs in six GEO datasets 
a

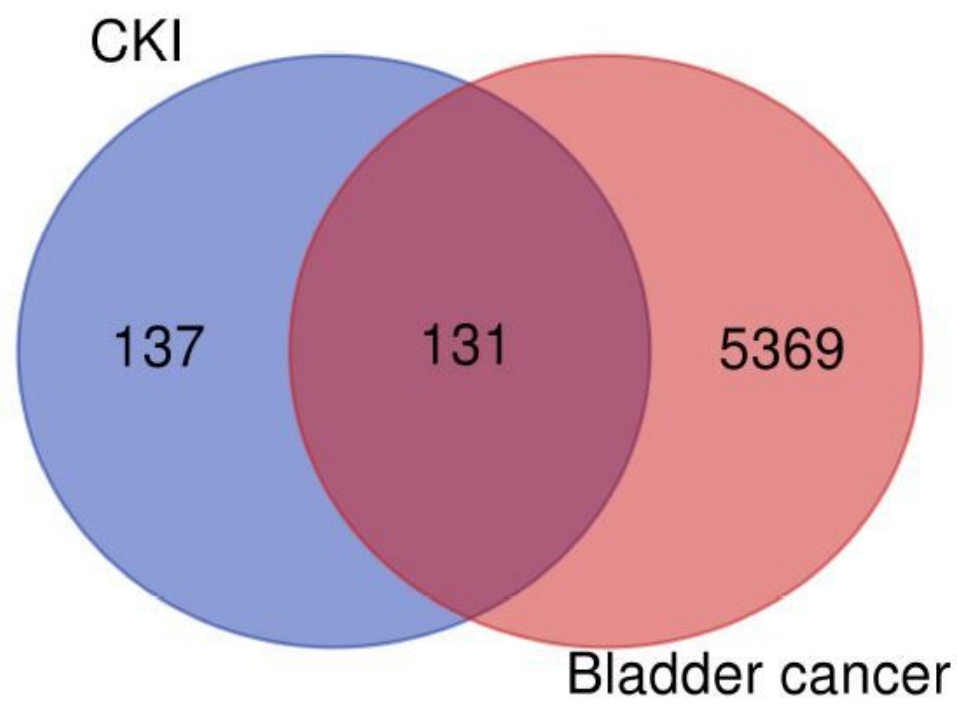

b

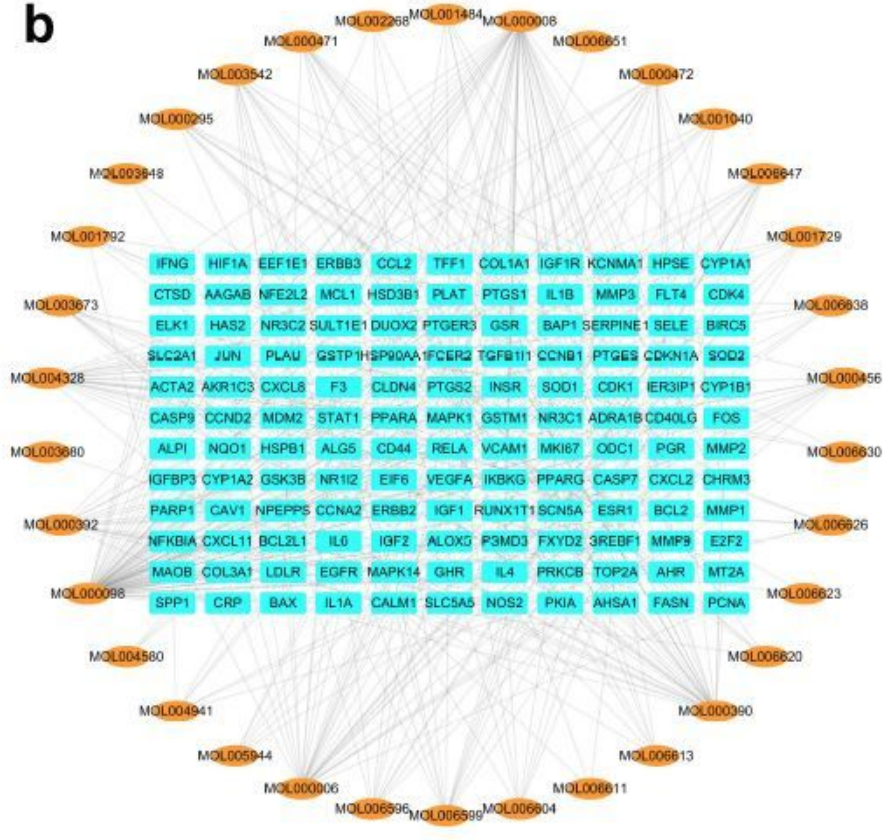

Figure 3

a Venn diagram of overlapping genes from CKI and DEGs. b Drug-target-disease network

a

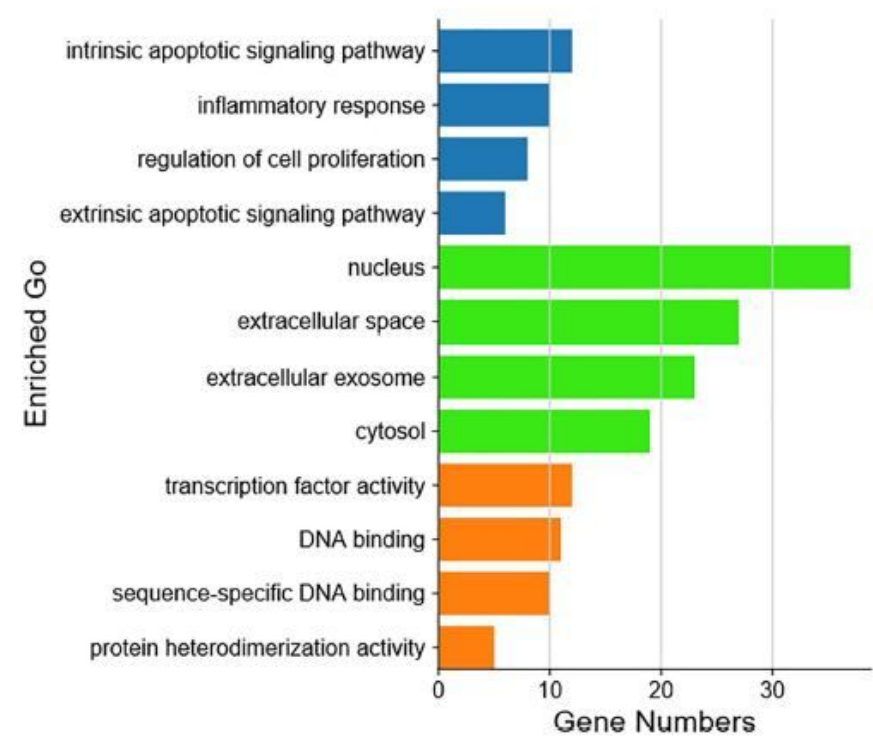

b

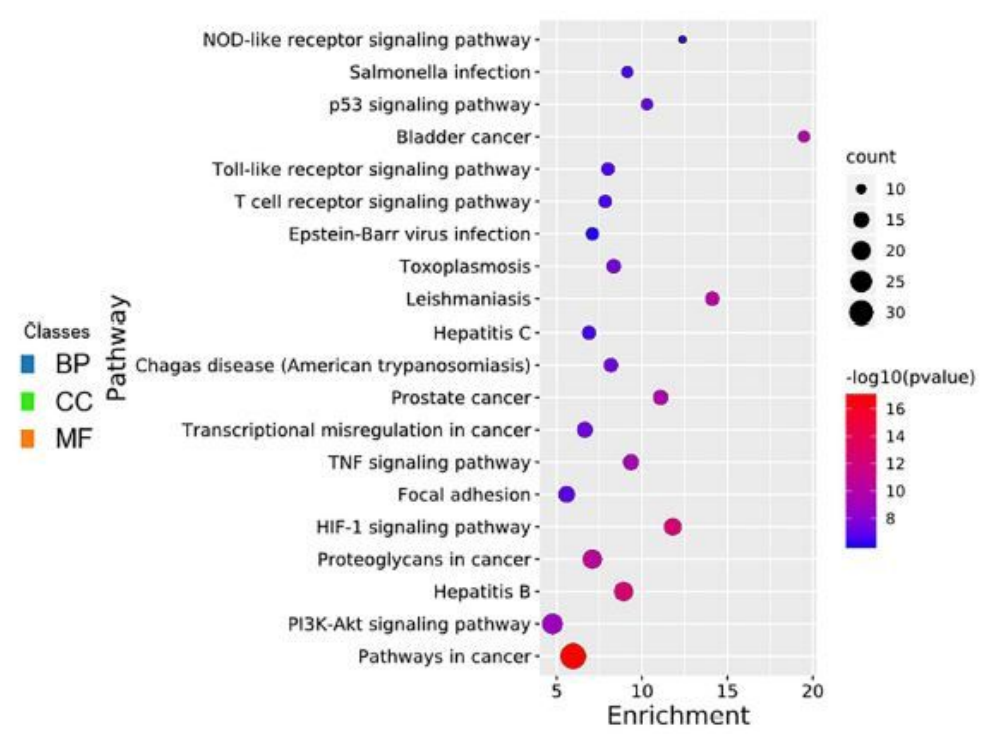

\section{Figure 4}

a GO enrichment analysis of common targets. The top four significantly enriched terms in BP, CC, and MF categories. $\mathrm{b}$ The top $20 \mathrm{KEGG}$ pathways identified by enrichment analysis of putative targets 
CYP1A2 RELA FCER2 - GSTP1 - ERBB2 - MMP1 SERPINE+ CALM1 - MMP2 - FXYD2 - MMP9 SLC5A5 IFNG DUOX2 ALPI MAOB PTGER3 CXCL8 MAPK14 CHRM 3 CRP BIRC5 EIF6 TOFBIII AHSA1 NOS2 ACTA2 NFKBIA IGF2 PSMO3 MAPK1 SLC2A1 PTGES IL1B COL3AF CXCL2 CLON4 CDK4 SCN5A IGF1R CCND2 NR112 NR3C2 NFE212 VCAM1 CCL2 SPP1 HSDSB1 HSPB1 E2F2 CASP9 CDK1 CDKN1A PKIA FASN F3 SREBF1 NR3C1 LOLR GSR HIFIA AKRIC3 INSR COLIAI OSK3B VEGFA CD44 HSP9OAA1 SOD2 AHR CASP7 CCNB1 TFF1 SELE SULTIE1 MK167 PPARG CXCL11 ALOXS IL1A TOP2A PTGS2 MCL1 FOS MMP3 NOO1 CAV1 FLT4 MOM2 CCNA2 BAX CYP1A1 PLAT PARP1 IGFBP3 KCNULAL STAT1 ADRA1B PRKCB RUNXIT1 JUN - OOC1 ELK1 EGFR PPARA CDAOLG IKBKG ESR1 12.4 ERBB3 CYP1B1 PTOS1 IGF1 CTSO SOD1 - PLAU - PCNA PGR - BAP1 BCL2 HAS2 NPEPPS MTZA ILE HPSE GSTMI GHR

a

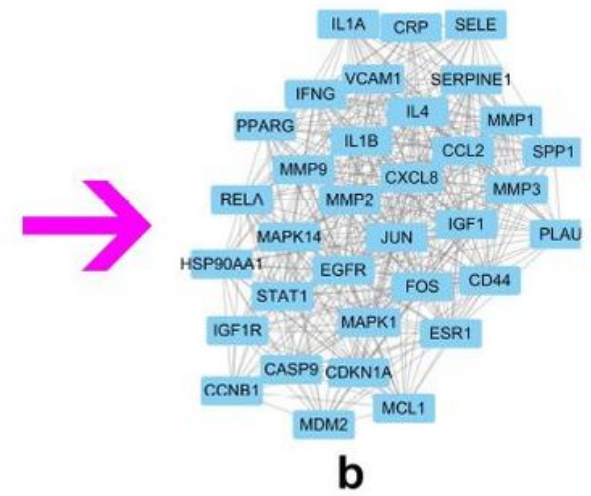

Figure 5

PPI network, module analysis, and hub gene identification

a
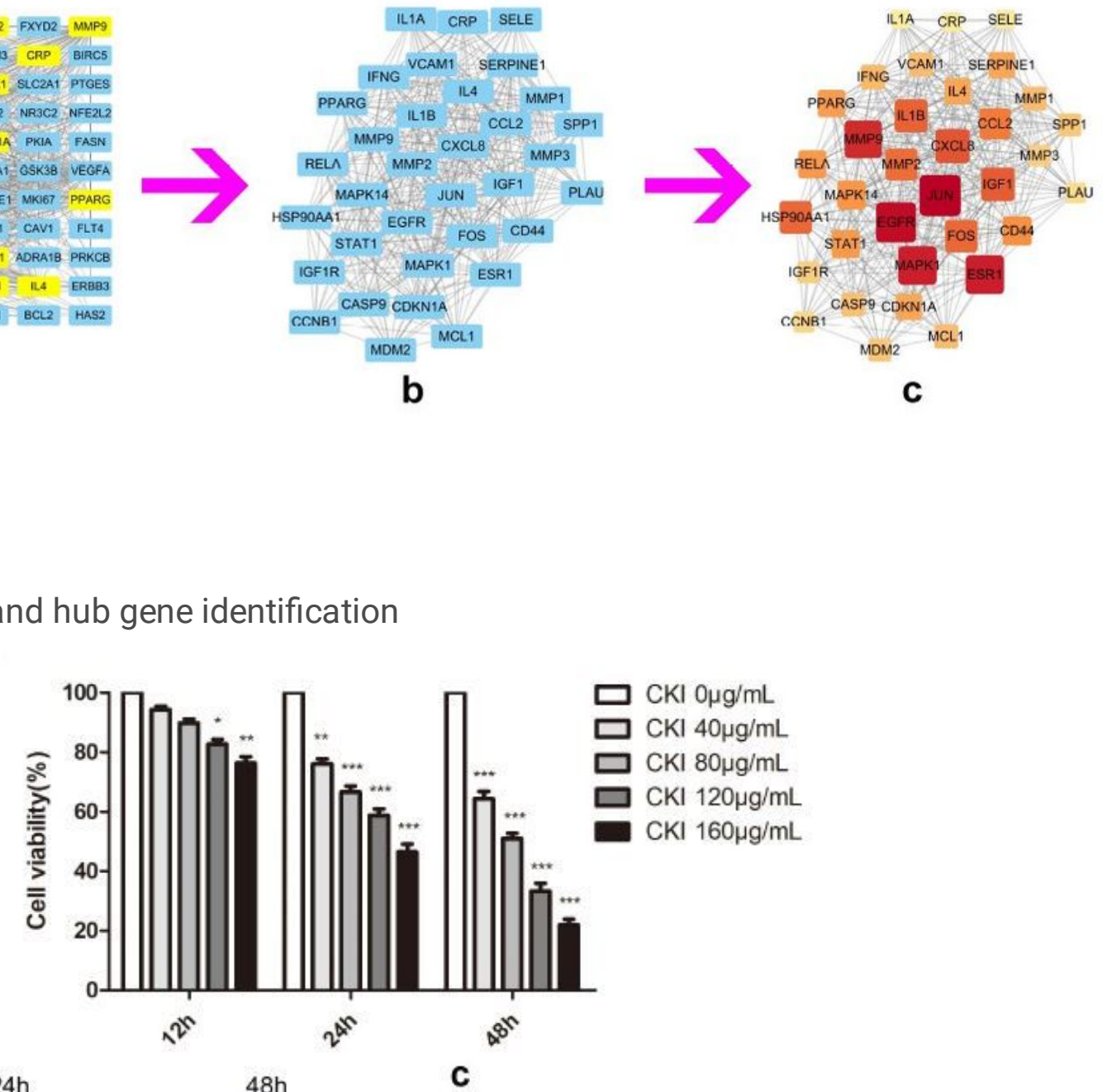

b

Oh

$24 \mathrm{~h}$

$48 h$
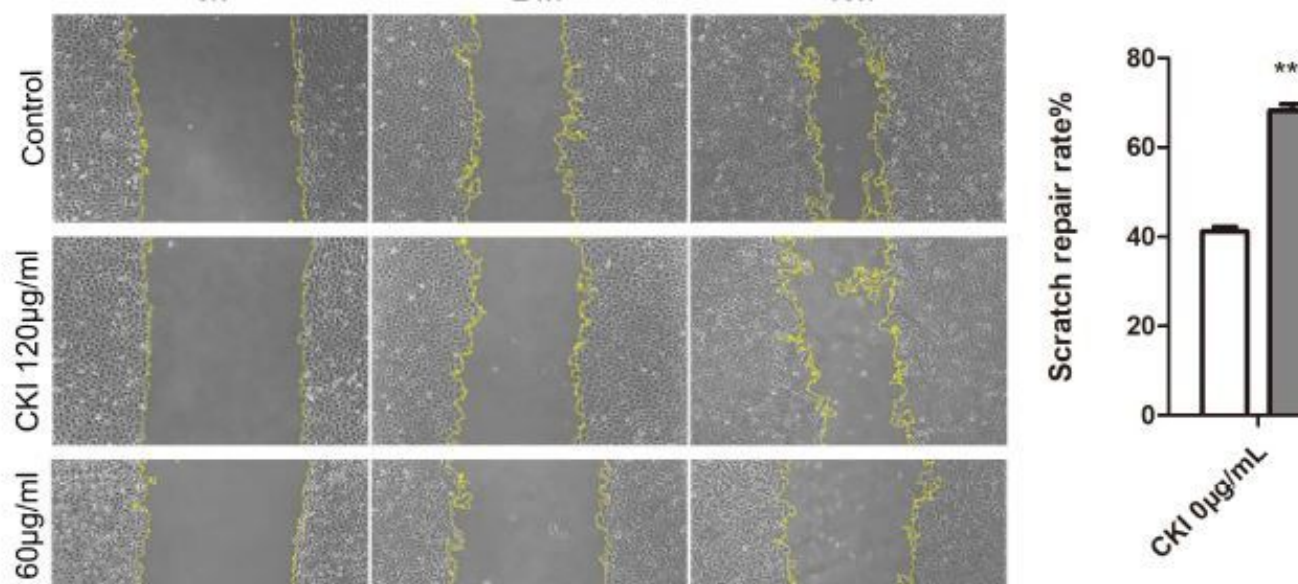

$\mathrm{CKI} 0 \mu \mathrm{g} / \mathrm{mL}$

CKI $40 \mu \mathrm{g} / \mathrm{mL}$

$\mathrm{CKI} 80 \mu \mathrm{g} / \mathrm{mL}$

$\mathrm{CKI} 120 \mu \mathrm{g} / \mathrm{mL}$

CKI $160 \mu \mathrm{g} / \mathrm{mL}$
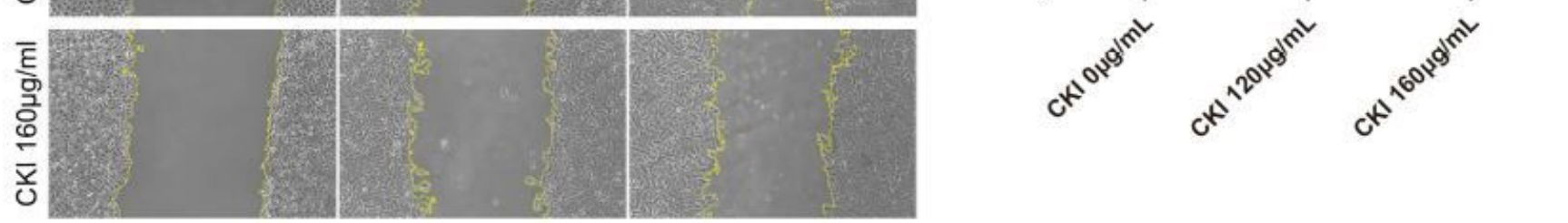

Figure 6

CKI significantly inhibited cell proliferation and migration. a The survival rate of T24 cells after treatment with CKI for 12, 24, and $48 \mathrm{~h}$. b Representative cell wound healing pictures. c Scratch repair rate of T24 cells after culture with $\mathrm{CKI}$ for 24 and $48 \mathrm{~h}$. ${ }^{*} \mathrm{P}<0.05, \star \star \mathrm{P}<0.01$ and $* \star * \mathrm{P}<0.001$ vs. control 

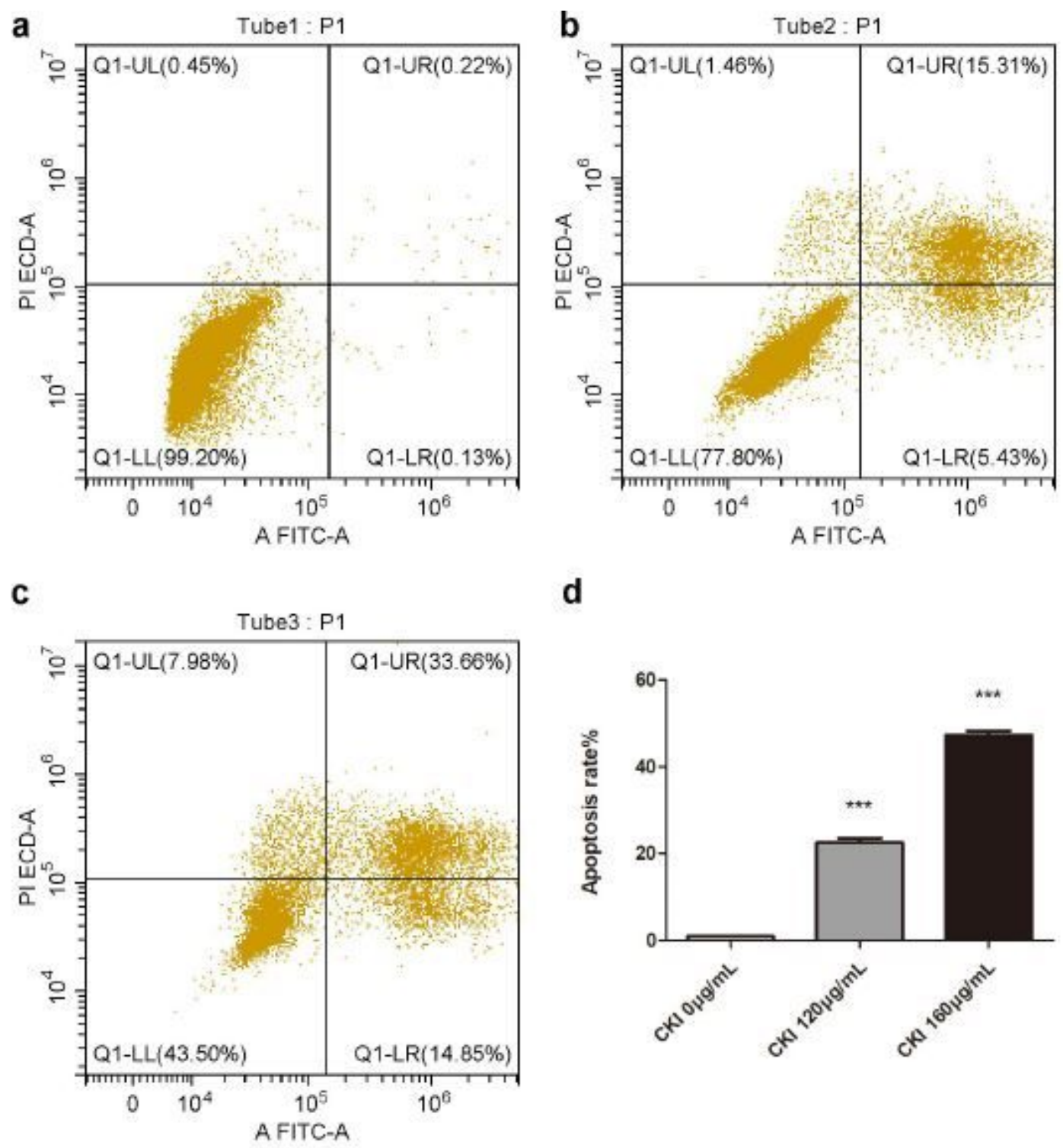

d

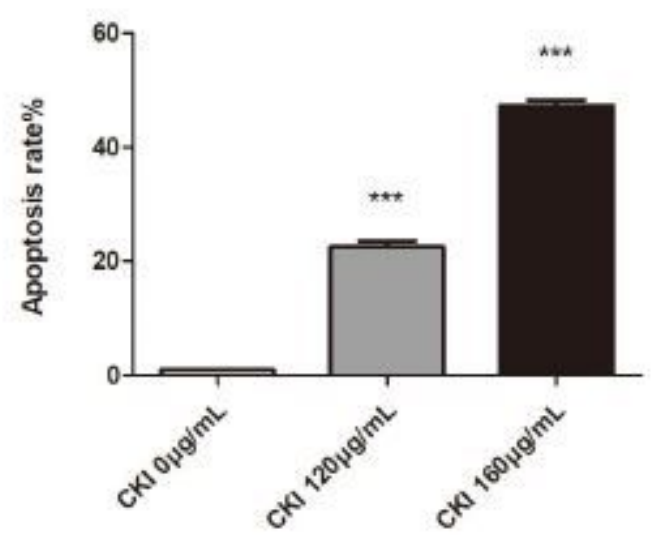

Figure 7

Flow cytometry was used to detect the apoptosis of T24 cells after treatment with CKI at concentrations of 0,120 , and $160 \mu \mathrm{g} / \mathrm{mL}$ for $24 \mathrm{~h}$ a, b, c. d Apoptotic rates increased in a dose-dependent manner. ${ }^{* \star *} \mathrm{P}<$ 0.001 vs. control 


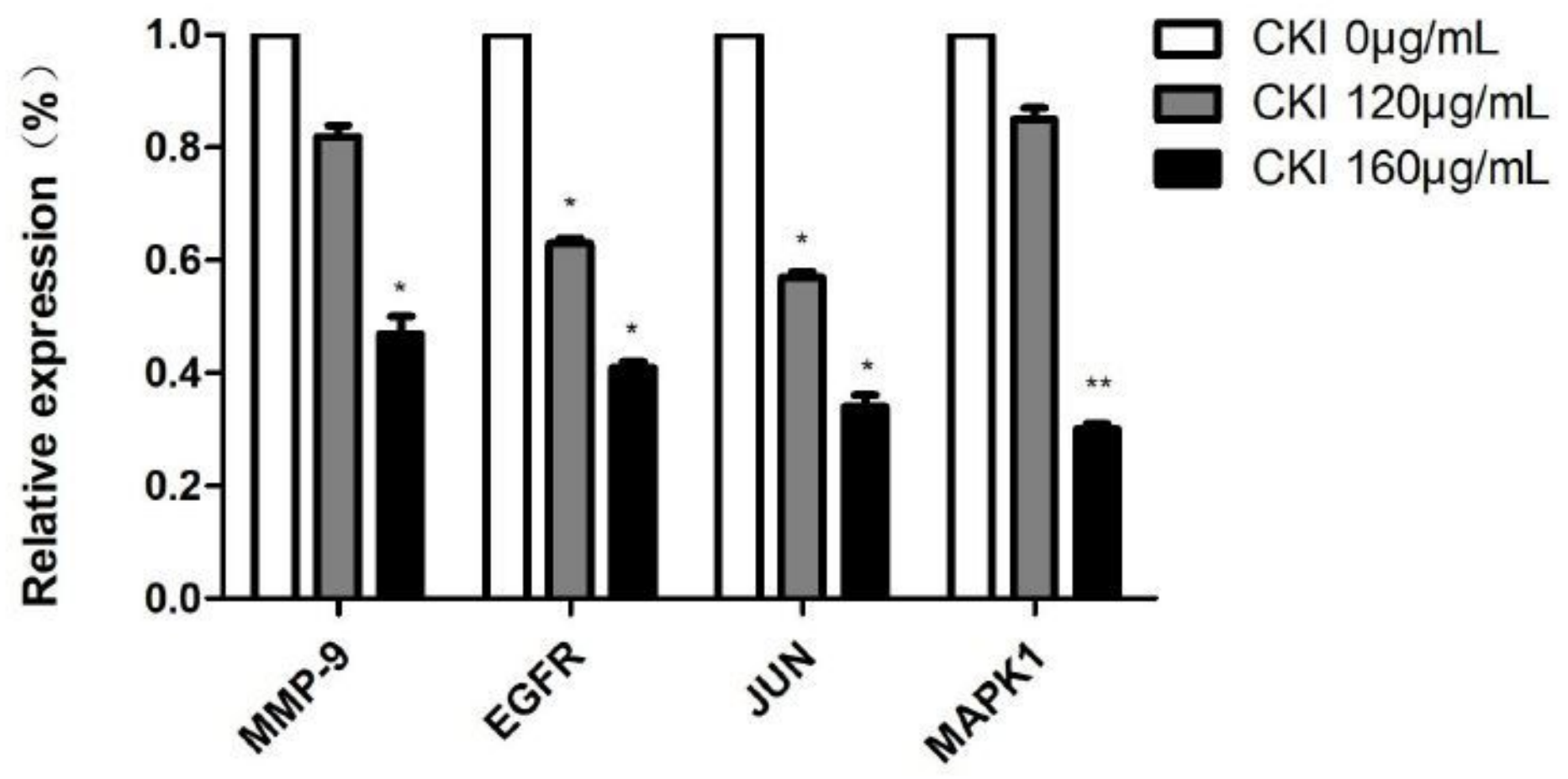

Figure 8

RT-qPCR of MMP-9, EGFR, JUN, and MAPK1 mRNA expression in T24 cells after treatment with CKI for 24 h. ${ }^{*} P<0.05,{ }^{*} \mathrm{P}<0.01$ vs. control 
a

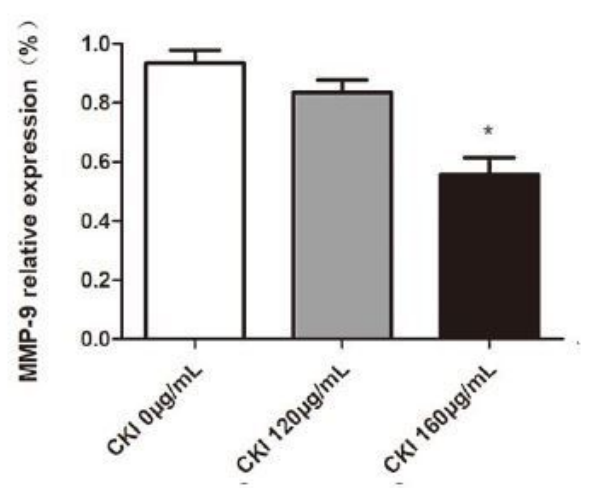

d

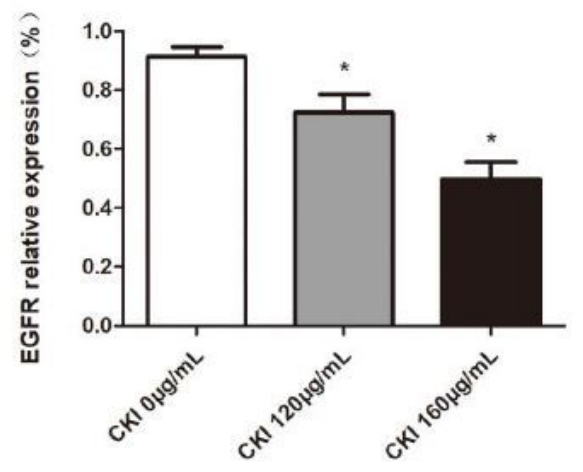

b

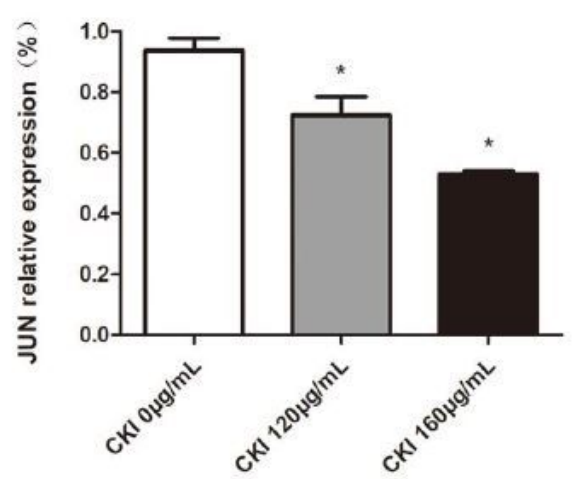

C

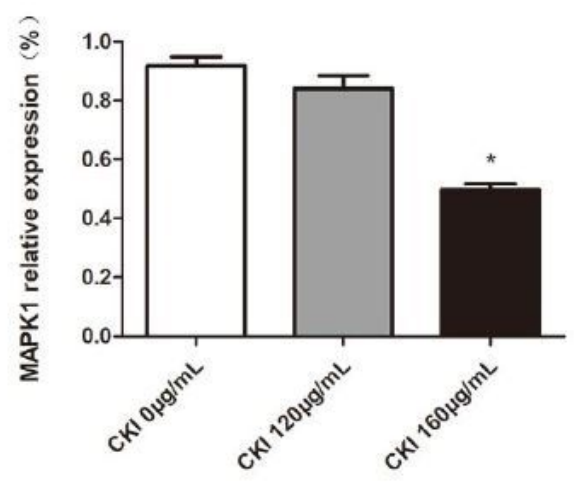

e

CKI 0 CKI $120 \quad$ CKI 160

MMP9

$78 \mathrm{KD}$

EGFR

69KD

JUN

43KD

MAPK1

43KD

$\beta$-actin

$42 \mathrm{KD}$

Figure 9

CKI can alter key protein expression levels in T24 cells. $\beta$-actin was used as an internal control. Protein expression levels of MMP-9 a, EGFR b, JUN c, and MAPK $1 \mathrm{~d}$ are visualized by statistical diagrams. e Representative images of protein bands. ${ }^{*} \mathrm{P}<0.05$ vs. control

\section{Supplementary Files}

This is a list of supplementary files associated with this preprint. Click to download.

- Additionalfiles.doc

- Additionalfiles.doc

- attachment.pdf

- attachment.pdf 